\title{
Targeting compensatory MEK/ERK activation increases JAK inhibitor efficacy in myeloproliferative neoplasms
}

\author{
Simona Stivala, ${ }^{1}$ Tamara Codilupi, ${ }^{1}$ Sime Brkic, ${ }^{1}$ Anne Baerenwaldt, ${ }^{1}$ Nilabh Ghosh, ${ }^{1}$ Hui Hao-Shen, ${ }^{1}$ Stephan Dirnhofer, ${ }^{2}$ \\ Matthias S. Dettmer, ${ }^{3}$ Cedric Simillion, ${ }^{4}$ Beat A. Kaufmann, ${ }^{1}$ Sophia Chiu, ${ }^{5}$ Matthew Keller, ${ }^{5}$ Maria Kleppe, ${ }^{5}$ Morgane Hilpert, ${ }^{1}$ \\ Andreas S. Buser, ${ }^{6}$ Jakob R. Passweg, ${ }^{6}$ Thomas Radimerski, ${ }^{7}$ Radek C. Skoda, ${ }^{1}$ Ross L. Levine, ${ }^{5,8}$ and Sara C. Meyer ${ }^{1,6}$ \\ 'Department of Biomedicine, University Hospital Basel and University of Basel, Basel, Switzerland. ²Department of Medical Genetics and Pathology, University Hospital Basel, Basel, Switzerland. \\ ${ }^{3}$ Department of Pathology and ${ }^{4}$ Department of BioMedical Research, University of Berne, Berne, Switzerland. ${ }^{5}$ Human Oncology and Pathogenesis Program, Memorial Sloan Kettering Cancer Center, New \\ York, New York, USA. ${ }^{6}$ Division of Hematology, University Hospital Basel, Basel, Switzerland. ${ }^{7}$ Novartis Institutes for Biomedical Research, Basel, Switzerland. ${ }^{8}$ Leukemia Service, Memorial Sloan Kettering \\ Cancer Center, New York, New York, USA.
}

Constitutive JAK2 signaling is central to myeloproliferative neoplasm (MPN) pathogenesis and results in activation of STAT, PIЗK/AKT, and MEK/ERK signaling. However, the therapeutic efficacy of current JAK2 inhibitors is limited. We investigated the role of MEK/ERK signaling in MPN cell survival in the setting of JAK inhibition. Type I and II JAK2 inhibition suppressed MEK/ERK activation in MPN cell lines in vitro, but not in Jak2V617F and MPLW515L mouse models in vivo. JAK2 inhibition ex vivo inhibited MEK/ERK signaling, suggesting that cell-extrinsic factors maintain ERK activation in vivo. We identified PDCFR $\alpha$ as an activated kinase that remains activated upon JAK2 inhibition in vivo, and PDGF-AA/PDGF-BB production persisted in the setting of JAK inhibition. PDGF-BB maintained ERK activation in the presence of ruxolitinib, consistent with its function as a ligand-induced bypass for ERK activation. Combined JAK/MEK inhibition suppressed MEK/ERK activation in Jak2V617F and MPLW515L mice with increased efficacy and reversal of fibrosis to an extent not seen with JAK inhibitors. This demonstrates that compensatory ERK activation limits the efficacy of JAK2 inhibition and dual JAK/MEK inhibition provides an opportunity for improved therapeutic efficacy in MPNs and in other malignancies driven by aberrant JAK-STAT signaling.

\section{Introduction}

Myeloproliferative neoplasms (MPNs) are hematologic stem cell disorders characterized by dysregulated JAK2 signaling. They present as chronic leukemias with excessive myeloid cell output, with polycythemia vera (PV) manifest as predominant erythrocytosis, essential thrombocythemia (ET) as pronounced thrombocytosis, and myelofibrosis (MF) with megakaryocyte expansion and progressive bone marrow fibrosis (1). MF is the most severe form of MPN, with limited life expectancy particularly in patients with high-risk genomic and clinical features (2). MPNs may transform to secondary acute myeloid leukemia (AML) with dismal prognosis, or progress to hematopoietic failure (1). The only curative therapy for MPNs is hematopoietic stem cell transplantation, but as MPNs

Related Commentary: p. 1519

\section{Authorship note: RLL and SCM are co-senior authors.}

Conflict of interest: TR was a Novartis employee when the study was conducted and holds Novartis stock. RCS has consulted for and received honoraria from Novartis, Shire and Baxalta. CS is a contractor of Roche. RLL is on the supervisory board of Qiagen and is a scientific advisor to Loxo, Imago, C4 Therapeutics, and Isoplexis, which each include an equity interest. He receives research support from and has consulted for Celgene and Roche, he has received research support from Prelude Therapeutics, and he has consulted for Incyte, Novartis, Morphosys, and Janssen. He has received honoraria from Lilly and Amgen for invited lectures and from Gilead for grant reviews. Copyright: () 2019 American Society for Clinical Investigation Submitted: November 20, 2017; Accepted: January 29, 2019. Reference information: / Clin Invest. 2019;129(4):1596-1611. https://doi.org/10.1172/JCI98785 most often affect the elderly, this option is restricted to a subset of patients with limited efficacy and significant toxicity (3). Thus, effective therapeutic options are critically needed based on new insight into the molecular processes driving MPNs.

Activated JAK2 signaling represents a central feature of all MPNs (4), and JAK2 inhibitors are in clinical use for the treatment of MPNs (5). JAK2 is an intracellular nonreceptor tyrosine kinase essential for hematopoiesis $(6,7)$. It represents the primary mediator of cytokine signaling from the thrombopoietin receptor MPL and the erythropoietin and granulocyte-macrophage CSF receptors. JAK2 phosphorylation and dimerization initiate activation of several signaling pathways, including the transcription factors STAT3 and STAT5, the phosphatidylinositol-3-kinase (PI3K)/AKT pathway, and the mitogen-activated protein kinase (MAPK) pathway involving MEK and ERK kinases, which promote cell proliferation, differentiation, and survival via multiple effectors (8). In MPNs, JAK2 signaling is constitutively activated by gain-of-function mutations in JAK2, such as JAK2V617F in $95 \%$ of PV and $50 \%-60 \%$ of MF and ET (9-12), or mutations in JAK2 exon 12 (13). Mutations in the thrombopoietin receptor $M P L$ (14) and in the chaperone protein calreticulin $(C A L R)$ were identified and converge on activation of MPL-JAK2 signaling (15-19). The JAK1/JAK2 inhibitor ruxolitinib is approved for patients with intermediate- and high-risk MF (20, 21) and as a second-line therapy for PV (22). Additional JAK inhibitors are in late-stage clinical development $(23,24)$. These clinical JAK2 inhibitors act as ATP mimetics and bind to JAK2 in the active conformation (so-called type I inhibition), blocking the catalytic 
activity with the activation loop in the DFG-in conformation with maintained JAK2 phosphorylation (25). They effectively reduce splenomegaly and constitutional symptoms and prolong survival in MPN patients $(20,21,26-28)$. However, type I JAK2 inhibitors are not able to induce meaningful molecular remissions in most patients $(26,29)$. In addition, spleen and symptom responses are lost after prolonged treatment in a substantial proportion of patients $(27,30)$ along with reactivation of JAK2 signaling on the molecular level (31). We have observed cross-resistance among several type I JAK2 inhibitors, including ruxolitinib, momelotinib, fedratinib, and BMS911543, in MPN cells by this mechanism (31-33). Thus, approaches to more effectively target activated JAK2/downstream signaling in MPNs are urgently needed.

The MAPK signaling pathway, here also referred to as the MEK/ERK pathway, engages 3 tiers of kinases, RAF, MEK1/2, and ERK1/2 (34), and is typically activated by the GTPase RAS. MAP kinases undergo sequential phosphorylation events and promote cell proliferation, differentiation, and survival via a multitude of cytoplasmic and nuclear effectors, including transcription factors, cell cycle regulators, kinases, and phosphatases (35). MEK1/2 represent the intermediate kinases in the MAPK pathway, while ERK1/2 are distal in the cascade. MEK1/2 are dual-specificity kinases phosphorylating tyrosine and threonine residues of ERK1/2, which represent their exclusive substrates (34). Combined ablation of ERK1 and ERK2 suppresses hematopoiesis, leading to cytopenias $(36,37)$. The MEK/ERK signaling pathway is frequently activated in solid tumors owing to hyperactive growth factor receptors or somatic mutations in RAS or RAF family members such as in melanoma or colorectal cancer (38). Mutations in NRAS or KRAS isoforms occur in AML, including in post-MPN AML (39), and the activating V600E mutation in $B R A F$ is characteristic of hairy cell leukemia (40). Inactivation of the tumor suppressor NF-1, a negative regulator of RAS, also results in constitutive MEK/ERK activation in myeloid malignancies $(37,41)$. In these RAS- and RAF-driven malignancies, the activation of the MEK/ERK signaling pathway is well understood, and several approaches have been studied in preclinical and clinical contexts to therapeutically target activated MEK/ ERK signaling, particularly by RAF or MEK1/2 inhibition (42-44). RAF inhibitors mediate clinical benefits in hairy cell leukemia and melanoma with specific mutations in $\operatorname{BRAF}(45,46)$, and combined RAF and MEK1/2 inhibition can provide further improved efficacy in these cancers $(47,48)$. However, RAF inhibition has been found to increase MEK/ERK signaling in tumors with wild-type RAF by promoting RAF dimerization and adaptive feedback signaling in this setting (48-50). Inhibitors of MEK1/2 such as trametinib, binimetinib, or selumetinib show a favorable profile with an overall good tolerability, as they target MEK1/2 via allosteric, non-ATPcompetitive binding, which enhances their specificity and reduces off-target inhibition of other kinases $(43,51)$.

The current insight into MEK/ERK signaling in solid tumors and certain leukemias provides the basis for therapeutic targeting of this pathway in these malignant contexts. In contrast, studies on the role of the MEK/ERK signaling pathway in MPNs are limited so far (52). Given that the impact of JAK2 inhibition on the MEK/ ERK signaling pathway in MPNs has not been studied in vivo, we hypothesized that MEK/ERK signaling could contribute to MPN cell survival in the setting of JAK inhibition. Here we address the role of the MEK/ERK signaling pathway in MPNs in the in vivo setting. We study whether compensatory MEK/ERK activation in the presence of JAK2 inhibition could contribute to the limited efficacy of JAK2 inhibitors in the clinic and whether combined JAK2 and MEK/ERK inhibition represents a superior therapeutic approach in MPN.

\section{Results}

ERK activation is inhibited by JAK2 inhibition in MPN cells, but not in vivo in JAK2V617F or MPLW515L mutant MPNs. Constitutive JAK2 activation by JAK2V617F and other MPN disease alleles mediates downstream activation of ERK1/2, the distal kinases in the MEK/ ERK pathway, as well as of STAT5, STAT3, and PI3K/AKT (12). In line with this notion and previous reports, we observed that JAK2 inhibition by the type I JAK2 inhibitor ruxolitinib promptly suppressed activation of ERK1/2 in the JAK2V617F mutant SET-2 cell line as reflected by reduced levels of phosphorylated ERK1/2 (p-ERK1/2) 30 minutes after initiation of exposure. The pronounced inhibition of ERK1/2 phosphorylation by ruxolitinib is maintained with prolonged exposure for 48 hours paralleled by reduced levels of p-STAT5 and p-STAT3 (Figure 1A and Supplemental Figure 1A; supplemental material available online with this article; https://doi.org/10.1172/JCI98785DS1). We observed that type II JAK2 inhibition with CHZ868 or BBT594 also inhibited ERK1/2 phosphorylation in a dose-dependent manner, although not as efficiently as type I JAK2 inhibition by ruxolitinib (Figure 1B and Supplemental Figure 1, B and C), supporting the notion that JAK2 inhibition by type I or type II binding consistently inhibits activation of the MEK/ERK pathway in MPN cells.

In contrast, we observed different signaling dynamics upon JAK2 inhibition in the in vivo setting. In a Jak2V617F mutant MPN mouse model (53), which expresses the V617F mutation in mouse Jak2 and is characterized by hyperactive JAK2 signaling, 3-5 oral doses of ruxolitinib at $60 \mathrm{mg} / \mathrm{kg}$ or of CHZ868 at $40 \mathrm{mg} / \mathrm{kg}$ suppressed STAT phosphorylation in primary hematopoietic cells as expected. However, there was no inhibitory effect on p-ERK1/2 (Figure 1, C and D, and Supplemental Figure 1D), suggesting maintained ERK activation in the presence of JAK2 inhibitors in the in vivo context. In the MPLW515L mutant MPN model (14, 54 ), which expresses a mutation in human MPL and is also characterized by hyperactive JAK2 signaling, ruxolitinib could not suppress ERK1/2 phosphorylation, at $60-\mathrm{mg} / \mathrm{kg}$ or $90-\mathrm{mg} / \mathrm{kg}$ doses (Figure 1E and Supplemental Figure 1D). Type II JAK2 inhibition by CHZ868 did not reduce ERK activation in primary mouse splenocytes in the MPLW515L model (Figure 1F and Supplemental Figure 1D). Whereas STAT phosphorylation was consistently suppressed by CHZ868 at all doses tested, ERK1/2 activation remained maintained in the presence of JAK2 inhibition in vivo (Supplemental Figure 1E).

ERK activation is inhibited by JAK2 inhibition in primary MPN cells ex vivo. To address whether maintained MEK/ERK pathway activation in the presence of JAK2 inhibitors relates to a cellintrinsic or -extrinsic mechanism, we exposed primary mouse MPN cells to JAK2 inhibition ex vivo (Figure $1 G$ ). Ruxolitinib at 0.5-2 $\mu \mathrm{M}$ for 6-8 hours was able to inhibit ERK activation ex vivo, such as in Jak2V617F mutant mouse splenocytes with reduced levels of p-ERK and suppressed STAT phosphorylation (Figure 1H and Supplemen- 
A

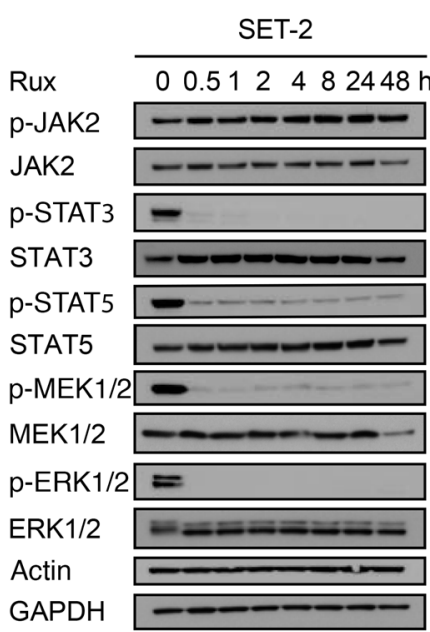

Type I
B

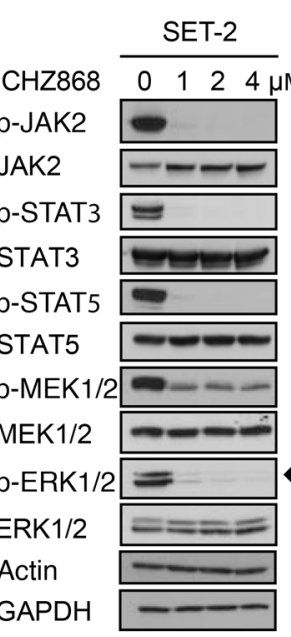

Type II
C

Rux

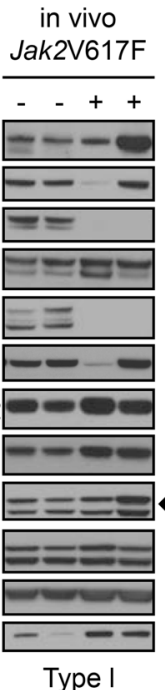

D

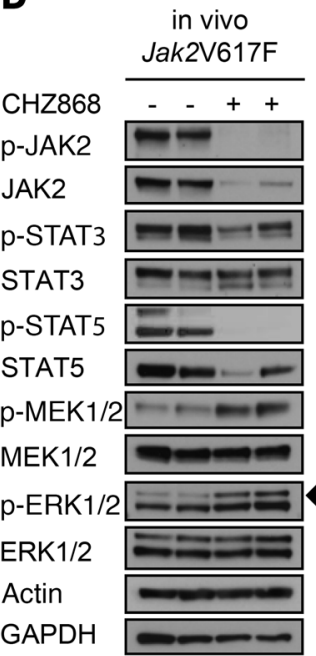

Type II
E

E MPLW515

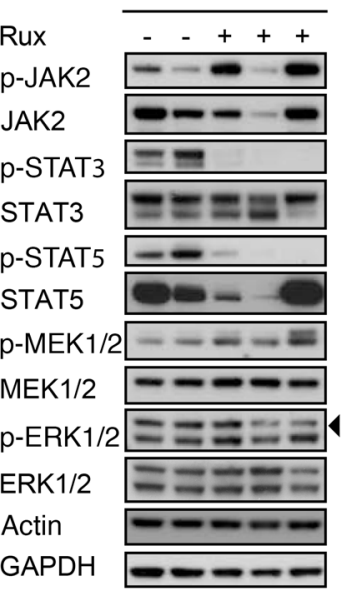

Type I
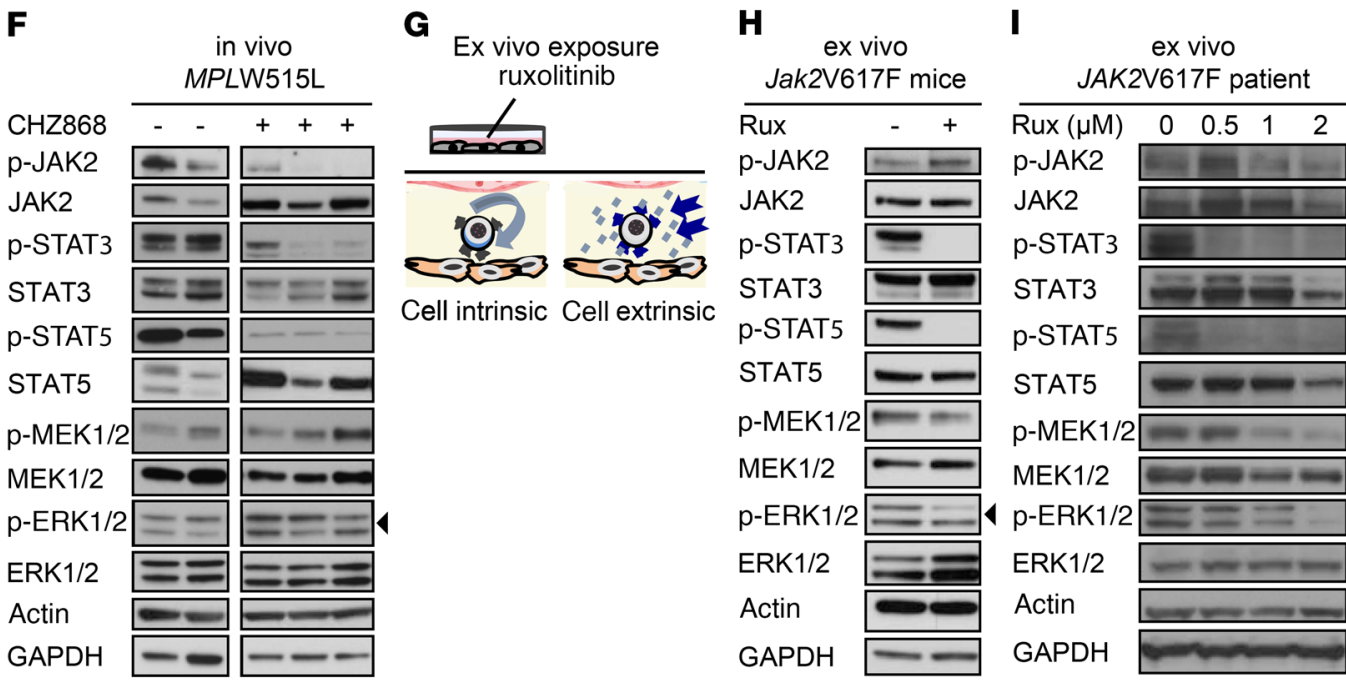

\section{J}

ex vivo MPLW515L mice

\begin{tabular}{ll}
\hline RUX & - \\
p-JAK2 & + \\
JAK2 & $=-$ \\
p-STAT3 & $=$ \\
STAT3 \\
p-STAT5 \\
STAT5 \\
p-MEK1/2 \\
MEK1/2 \\
p-ERK1/2 \\
ERK1/2 \\
Actin \\
GAPDH
\end{tabular}

Type II

Figure 1. ERK activation is maintained in the presence of JAK2 inhibition in vivo, but inhibited ex vivo. (A) Type I JAK2 inhibition by ruxolitinib at $1 \mu M$ inhibits STAT and ERK phosphorylation promptly and over 48 hours in JAK2V617F SET-2 cells $(n=4)$. (B) Type II JAK2 inhibition by CHZ868 at 1-4 $\mu$ M for 4 hours dose-dependently inhibits STAT and ERK phosphorylation in JAK2V617F SET-2 cells $(n=3)$. (C and D) Type I JAK2 inhibitor ruxolitinib at $60 \mathrm{mg} / \mathrm{kg}$ $(n=6)$ and type II JAK2 inhibitor CHZ868 at $40 \mathrm{mg} / \mathrm{kg}(n=6)$ administered orally to primary Jak2V617F mice for 3-5 doses inhibit STAT phosphorylation, whereas ERK phosphorylation is fully maintained in comparison with vehicle-treated mice $(n=6-7)$. (E and F) Type I JAK2 inhibitor ruxolitinib at $90 \mathrm{mg} / \mathrm{kg}$ $(n=16)$ and type II JAK2 inhibitor CHZ868 at $40 \mathrm{mg} / \mathrm{kg}(n=6)$ administered orally to MPLW515L transplanted mice for 3-5 doses inhibit STAT phosphorylation, whereas ERK phosphorylation remains fully maintained in comparison with vehicle-treated mice $(n=8-13)$. (G) Schematic of ex vivo experiments. Primary MPN mouse splenocytes were exposed to ruxolitinib at 0.5-2 $\mu \mathrm{M}$ for 6-8 hours to differentiate cell-intrinsic versus cell-extrinsic mechanisms of JAK2-independent ERK activation. (H) Ruxolitinib at $2 \mu \mathrm{M}$ inhibited STAT and ERK phosphorylation in primary Jak2V617F splenocytes upon ex vivo exposure for 8 hours $(n=4)$. (I) Ruxolitinib at $0.5-2 \mu \mathrm{M}$ dose-dependently inhibited STAT and ERK phosphorylation upon 8 hours of ex vivo exposure in PBMCs of JAK2V617F mutant MPN patients $(n=2)$. (J) Ruxolitinib at $0.5 \mu \mathrm{M}$ inhibited STAT and ERK phosphorylation upon ex vivo exposure in splenocytes of MPLW515L transplanted mice for 6 hours $(n=9)$ as compared with vehicle-treated mice $(n=8)$.

tal Figure 1H). ERK1/2 activation was dose-dependently inhibited in peripheral blood mononuclear cells (PBMCs) of a JAK2V617F mutant MPN patient exposed to ruxolitinib ex vivo (Figure 1I and Supplemental Figure 1H). Another JAK2V617F mutant MPN patient on long-term ruxolitinib treatment (20 $\mathrm{mg}$ twice daily) showed maintained ERK activation in freshly isolated peripheral blood PBMCs. By contrast, ex vivo ruxolitinib exposure reduced ERK activation in the context of systemic ruxolitinib treatment (Supplemental Figure 1, F and H). We exposed primary MPLW515L mutant mouse splenocytes to JAK2 inhibition by ruxolitinib ex vivo (Figure 1J). Consistent with the findings in the JAK2V617F mutant setting, ex vivo ruxolitinib exposure inhibited ERK phosphorylation in the context of the MPL mutation (Figure 1J and Supplemental Figure 1, $\mathrm{G}$ and $\mathrm{H}$ ), but not in vivo (Figure 1, E and F, and Supplemental Figure 1D). These data suggest that cell-extrinsic signals mediate ERK activation in the presence of JAK2 inhibition.

PDGFR $\alpha$ is activated in Jak2V617F and MPLW515L MPN models and not inhibited by JAK2 inhibitors along with maintained 
PDGF levels. To identify ligand/receptor activation cascades that could mediate JAK2-independent ERK activation in vivo, we performed receptor tyrosine kinase (RTK) arrays in Jak2V617F and MPLW515L mouse splenocytes in the presence and absence of in vivo ruxolitinib therapy. Whereas wild-type C57BL/6 mouse splenocytes showed activation of a multitude of RTKs, Jak2V617F mice primarily showed specific activation of PDGFR $\alpha$ (Figure 2A and Supplemental Figure 2A). PDGFR $\alpha$ activation was unaffected and maintained in the setting of JAK2 inhibitor treatment as assessed by RTK array (Figure 2A) and by conventional Western blotting (Figure 2B). Pdgfra mRNA expression was not significantly changed upon ruxolitinib treatment in bone marrow (BM) (Figure 2C) and spleen (Figure 2D) of Jak2V617F mice. MPLW515L mutant mice showed an analogous pattern with PDGFR $\alpha$ activation in the presence and absence of JAK2 inhibitor treatment (Supplemental Figure 2B). These data suggest that PDGFR $\alpha$ could mediate compensatory, JAK2-independent MEK/ERK pathway activation in vivo.

As a complementary approach to identify factors mediating compensatory ERK activation, we performed multiplexed RNA expression analyses in BM and spleen of the levels of 34 secreted ligands. This panel included cytokines previously implicated in the inflammatory milieu of MPNs $(55,56)$ and growth factors signaling through RTKs. By Luminex analyses, 31 soluble factors were assessed on the protein level in the BM and spleen interstitial fluid and in the serum of Jak2V617F mutant mice. The production of multiple cytokines was reduced by ruxolitinib treatment as previously reported (55). We focused on the cytokines/growth factors not suppressed by JAK2 inhibitor treatment, which represent potential mediators of JAK2-independent ERK activation. We observed that upon ruxolitinib treatment, levels of Pdgfa and $P d g f b$, which both signal through PDGFR $\alpha$, were maintained at the mRNA expression level in BM and spleen (Figure 2, C and D) as well as at the protein level in $\mathrm{BM}$ and spleen interstitial fluid in Jak2V617F mice (Supplemental Figure 2, C-G). Analysis in the $M P L W 515 \mathrm{~L}$ model confirmed this finding for Pdgfa (Supplemental Figure $2 \mathrm{H}$ ). Notably, among all the soluble factors not suppressed by ruxolitinib, PDGFs were the only cytokines maintained in all contexts, i.e., in BM and spleen, at an mRNA and protein level, in Jak2V617F and MPLW515L models (Supplemental Figure 2E), highlighting a potential role of the PDGF/PDGFR $\alpha$ axis as a mediator for compensatory ERK activation.

PDGF-BB/PDGFRa signaling bypasses JAK2 inhibition and mediates JAK2-independent ERK activation. As both PDGF-AA and PDGF-BB can signal through PDGFR $\alpha$ (57), we investigated their relative role in JAK2-independent MAPK activation. We determined PDGF-AA and PDGF-BB levels in BM and spleen interstitial fluid as well as serum of Jak2V617F mice and found that PDGF-BB concentrations were 2.5- to 4-fold higher than the levels of PDGFAA (Figure 2E). PDGF-BB levels also exceeded PDGF-AA in BM and spleen 2.5- to 6-fold when Jak2V617F mice were treated with ruxolitinib (Figure 2F). We then asked whether PDGF-BB could activate ERK1/2 in the presence of JAK2 inhibition in Jak2V617F mice ex vivo. When we stimulated Jak2V617F primary mouse BM cells with PDGF-AA or with PDGF-BB, in the presence of $1 \mu \mathrm{M}$ ruxolitinib, PDGF-BB was able to maintain p-ERK1/2 (Figure 2G). ERK activation was even more pronounced when PDGF-BB ex vivo stimulation was preceded by ruxolitinib pretreatment for 6 hours, leading to 3-fold increased p-ERK1/2 levels (Figure 2H). In contrast, stimulation with PDGF-AA ex vivo did not activate ERK in the presence of ruxolitinib in Jak2V617F BM cells. Similar to the effects in Jak2V617F BM, PDGF-BB activated ERK signaling in spleen cells as reflected by increased p-ERK levels, but to a lesser extent than in Jak2V617F BM (Supplemental Figure 3A). To investigate the cell populations responsible for the production of PDGF-BB mediating compensatory ERK activation, we assessed PDGFB expression in sorted BM progenitor and differentiated hematopoietic cells of Jak2V617F mice. Upon ruxolitinib treatment, we observed significantly increased $P d g f b$ expression in megakaryocyte-erythroid progenitors (MEPs), a population typically expanded in MPNs, along with a more modest, nonsignificant increase in Pdgfb expression in common myeloid progenitor (CMP) cells (Supplemental Figure 3B). Pdgfra expression was enhanced less extensively in hematopoietic progenitor compartments, including MEP, CMP, GMP, and LSK, as well as in mature granulocytes and lymphocytes, by ruxolitinib treatment (Supplemental Figure 3C). Pdgfb expression was 8- to 10-fold higher than Pdgfa expression in both MEPs and CMPs (Figure 2, I and J). Overall, these findings demonstrate PDGF/PDGFR $\alpha$ signaling as a mediator of JAK2-independent ERK activation in MPNs with PDGF-BB as the primary ligand mediating this effect.

Combined JAK2 and MEK inhibition prevents ERK activation and provides superior therapeutic efficacy in a Jak2V617F MPN mouse model. We next investigated whether targeting JAK2independent MEK/ERK pathway activation would provide a therapeutic benefit. We chose to target MEK1/2, the intermediate kinases in the MEK/ERK pathway. MEK1/2 inhibitors are allosteric, non-ATP-competitive inhibitors with a relatively narrow specificity spectrum. In contrast to ERK inhibitors, which are less well characterized, several MEK inhibitors have been clinically tested in other malignancies with favorable efficacy and tolerability profiles as both single-agent and combination therapies $(51,58)$. We first explored the effects of combined JAK2/MEK inhibition on signaling in JAK2V617F mutant SET-2 cells using ruxolitinib plus binimetinib, a MEK inhibitor in advanced clinical development. When SET-2 cells were exposed to the respective inhibitors for 4 hours, ruxolitinib dose-dependently suppressed STAT3, STAT5, and ERK phosphorylation in line with our previous experiments (Figure 1A) and with the notion that ERK activation in MPNs is JAK2-dependent. Binimetinib resulted in dosedependent ERK inhibition without inhibitory effects on STAT3 and STAT5, while combined ruxolitinib and binimetinib inhibited ERK activation to a greater extent than ruxolitinib or binimetinib monotherapy (Supplemental Figure 4).

To demonstrate the therapeutic potential of combined JAK2 and MEK/ERK pathway inhibition in a relevant in vivo context, we next investigated the impact of combined JAK/MEK inhibition on the Jak2V617F model of PV (53). We treated recipients engrafted with both Jak2V617F CD45.2 and Jak2 wild-type CD45.1 BM. We observed suppressed ERK1/2 and STAT5 activation when mice were treated with combined ruxolitinib $60 \mathrm{mg} / \mathrm{kg}$ twice daily (b.i.d.) and binimetinib at 10, 20, or $30 \mathrm{mg} / \mathrm{kg}$ b.i.d. (Figure $3 \mathrm{~A}$ ). Ruxolitinib significantly reduced splenomegaly in line with previous reports, while combined ruxolitinib plus binimetinib at $30 \mathrm{mg} / \mathrm{kg}$ b.i.d. normalized spleen size upon 4 weeks of treatment (Figure 3B). Notably, binimetinib monotherapy also reduced splenomegaly, albeit 
A
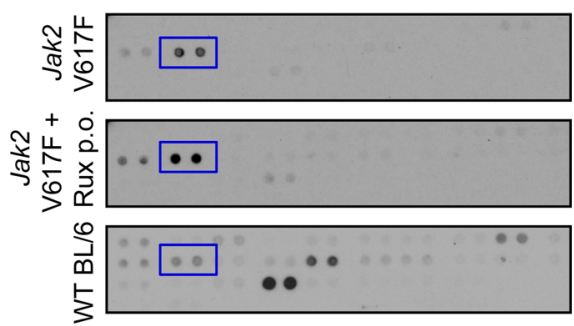

B

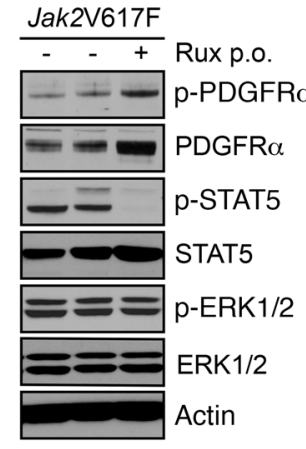

C

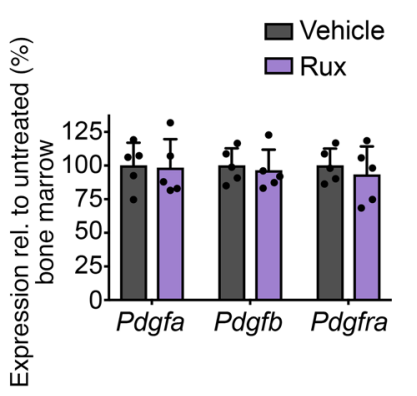

D

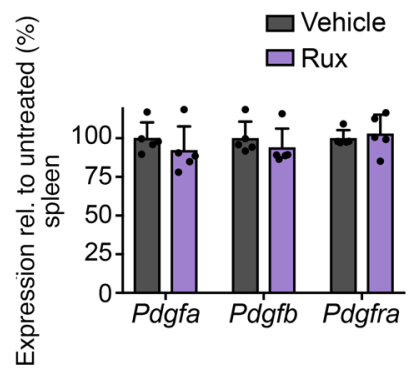

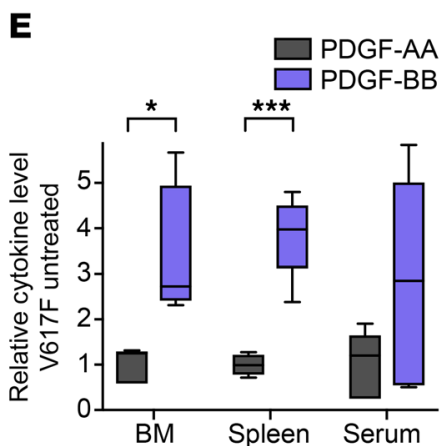

$\mathbf{F}$

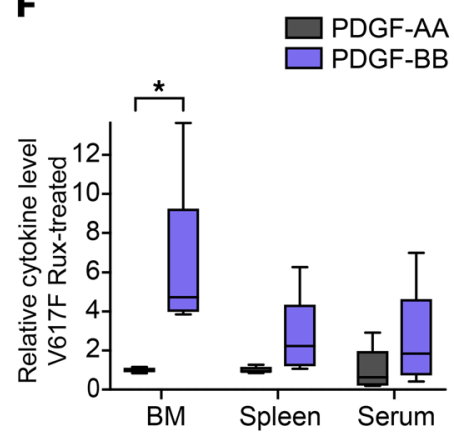

G

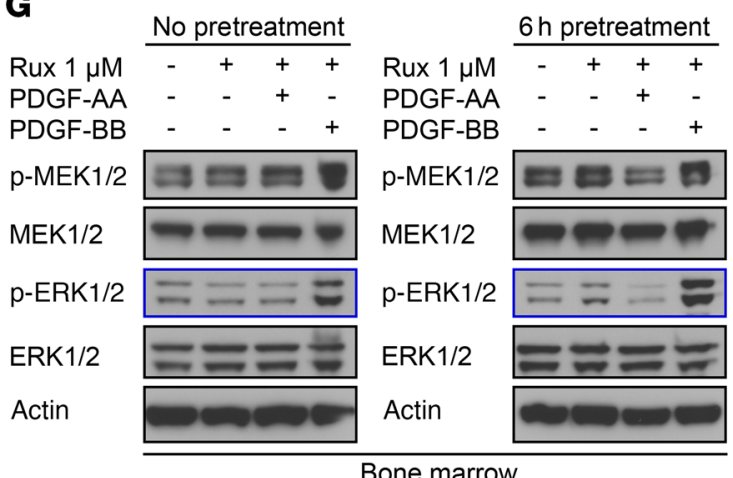

H

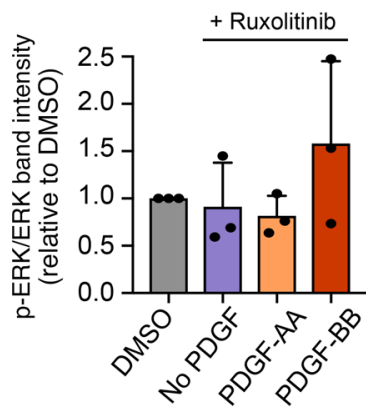

I

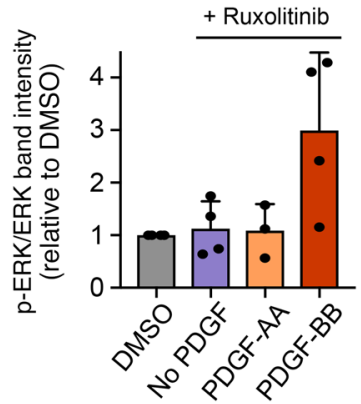

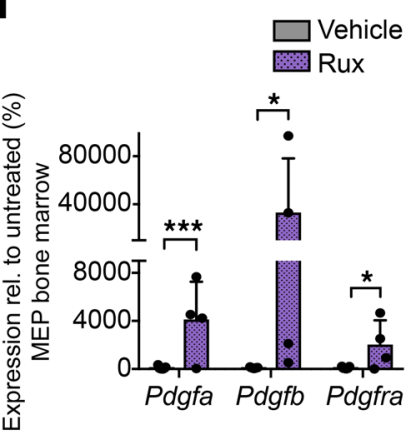

J

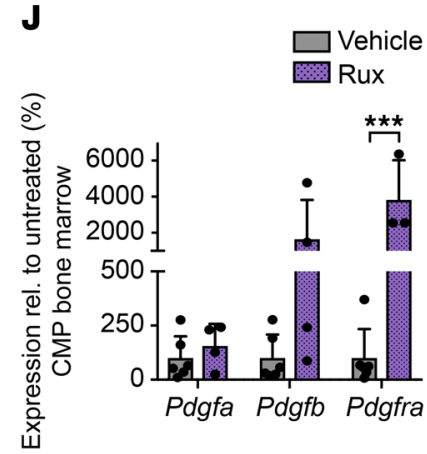

Figure 2. PDGF-BB-PDGFR $\alpha$ signaling bypasses JAK2 inhibition, mediating JAK2-independent ERK activation. (A) RTK arrays of Jak2V617F splenocytes treated with vehicle (top) or ruxolitinib (middle) show primarily activation of PDGFR $\alpha$ (blue, in duplicate) versus C57BL/6 (bottom, $n=1-2$ per condition). Additional RTKs in the array are specified in Supplemental Figure 2, A and B. (B) PDGFR $\alpha$ remains activated in Jak2V617F mouse splenocytes upon $60 \mathrm{mg} / \mathrm{kg}$ ruxolitinib ( $n=1-2$ per condition). (C) Expression of Pdgfa, Pdgfb, and Pdgfra in Jak2V617F mouse BM is maintained upon $60 \mathrm{mg} / \mathrm{kg}$ ruxolitinib ( $n=5$ per group). (D) Expression of Pdgfa, Pdgfb, and Pdgfra in Jak2V617F mouse splenocytes is maintained upon $60 \mathrm{mg} / \mathrm{kg}$ ruxolitinib ( $n=5$ per group). (E) PDCF-BB levels are higher than PDGF-AA levels in BM, spleen interstitial fluid, and serum of untreated Jak2V617F mice ( $n=5$ per group). (F) PDCF-BB levels are higher than PDCF-AA levels in BM, spleen interstitial fluid, and serum of Jak2V617F mice upon ruxolitinib $60 \mathrm{mg} / \mathrm{kg}$ ( $n=5$ per group). (C) Jak2V617F mouse BM was exposed ex vivo to ruxolitinib $1 \mu \mathrm{M}$ and PDGF-AA $100 \mathrm{ng} / \mathrm{ml}$ or PDGF-BB $200 \mathrm{ng} / \mathrm{ml}$ without (left) or with (right) ruxolitinib pretreatment for 6 hours. PDGF-BB maintained ERK phosphorylation in the presence of ruxolitinib. (H) Densitometries confirm increased ERK phosphorylation by PDCF-BB in the presence of ruxolitinib in Jak2V617F BM ex vivo ( $n=3-4)$. (I) Pdgfa, Pdgfb, and Pdgfra expression levels in megakaryocyte-erythroid progenitors (MEPs) of Jak2V617F BM are elevated upon ruxolitinib treatment ( $n=4-7$ per group). (J) Pdgfa, Pdgfb, and Pdgfra expression levels in common myeloid progenitors (CMPs) of Jak2V617F BM are elevated upon ruxolitinib treatment ( $n=3-6$ per group). Quantitative results were analyzed by 1-way ANOVA with $P \leq 0.05$ considered significant and are shown as mean $\pm \mathrm{SD}$ (C, D, $\mathbf{H}-\mathbf{J})$ or as medians with boxes representing 25th to 75th percentiles and whiskers indicating minimum and maximum values (E and $\mathbf{F}) .{ }^{*} P<0.05,{ }^{* * *} P<0.005$.

to a lesser extent than JAK2 inhibition, in line with a relevant role of MEK/ERK pathway activation in MPN pathogenesis (Figure 3B). Hematocrit was substantially elevated in untreated mice, reflecting the polycythemia phenotype, and ruxolitinib monotherapy was not able to substantively reduce hematocrit within 4 weeks of treatment. However, combined ruxolitinib/binimetinib significantly decreased hematocrit to the normal range with just 4 weeks of treat- ment (Figure 3, C and D). Reticulocytes were significantly lowered by combined therapy (Figure $3 \mathrm{E}$ ). Both $\mathrm{Lin}^{-} \mathrm{Sca1}^{-} \mathrm{Kit}^{+}$multipotent myeloid progenitors and $\mathrm{CD}_{71}{ }^{+}$erythroid progenitors, which are typically expanded in the Jak2V617F model, were reduced by ruxolitinib and binimetinib monotherapy, while combined JAK and MEK inhibition achieved significantly superior reduction of both populations (Figure 3, F and G). The proportion of $\mathrm{Lin}^{-} \mathrm{Sca1}{ }^{-} \mathrm{Kit}^{+} \mathrm{CD} 41^{-}$ 
A

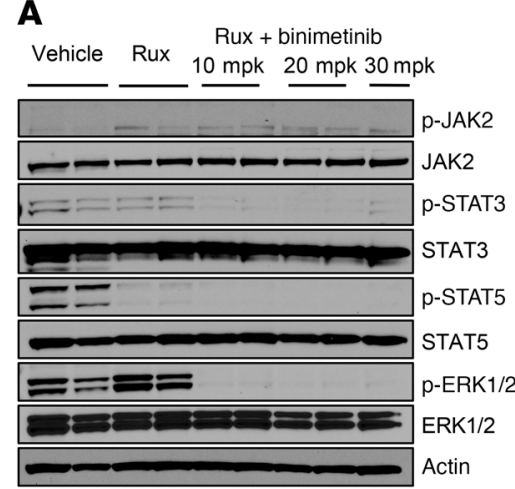

D

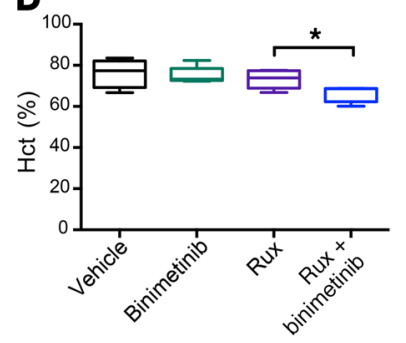

H

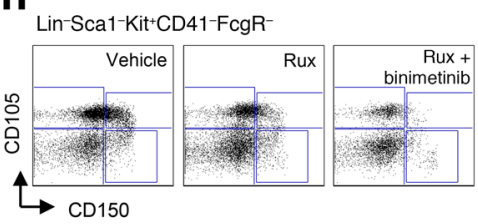

B
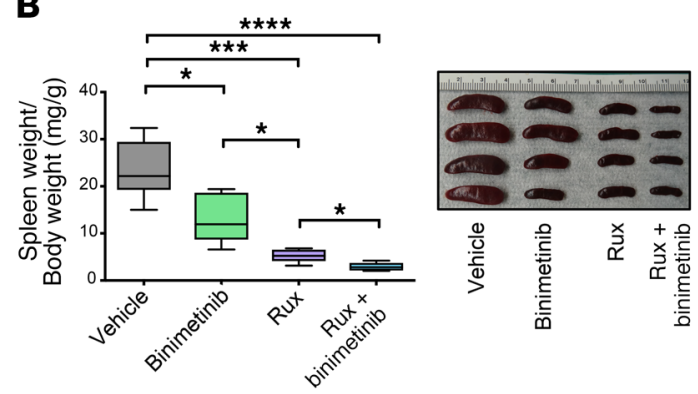

E

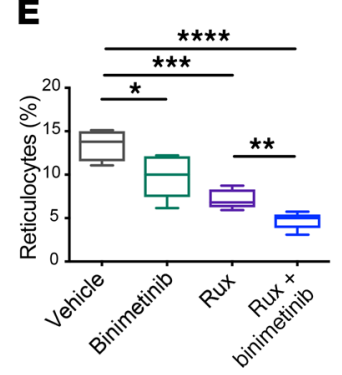

$\mathbf{F}$

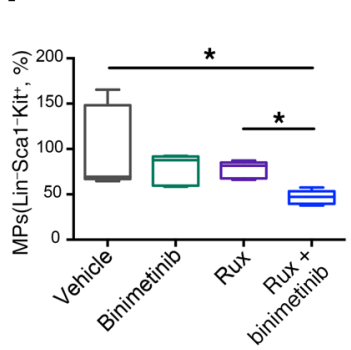

C

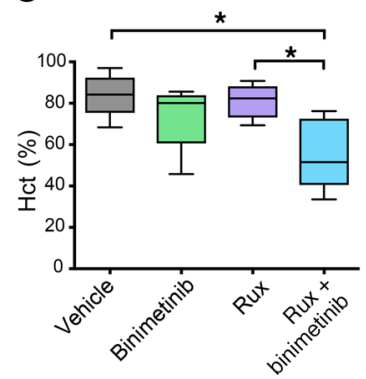

G
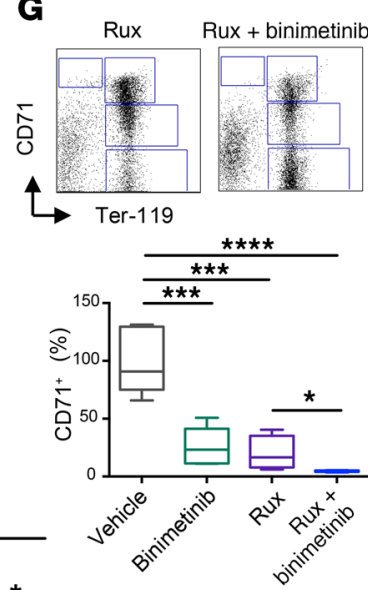
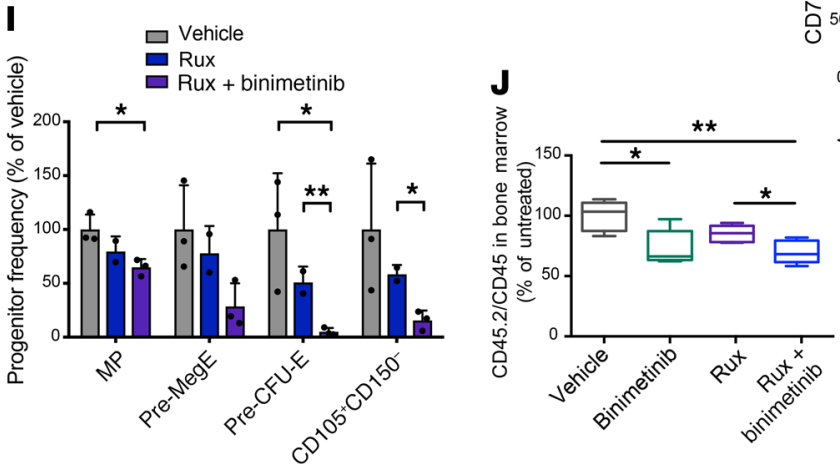

Figure 3. Combined JAK2 and MEK inhibition provides superior therapeutic efficacy in a Jak2V617F MPN mouse model. (A) Combined ruxolitinib 60 $\mathrm{mg} / \mathrm{kg}$ and binimetinib $10-30 \mathrm{mg} / \mathrm{kg}$ for 1 week completely inhibited STAT and ERK phosphorylation in Jak2V617F splenocytes, whereas ruxolitinib alone resulted in maintained ERK activation. (B) Binimetinib $30 \mathrm{mg} / \mathrm{kg}$ for 4 weeks moderately reduced splenomegaly, although to a lesser extent than ruxolitinib $60 \mathrm{mg} / \mathrm{kg}$. Combined binimetinib/ruxolitinib showed a superior effect compared with either single agent. (C) Combined binimetinib/ruxolitinib for 4 weeks reduced the elevated hematocrit (Hct), which was not seen with either monotherapy. (D) Hematocrit was only slightly reduced after combined binimetinib/ruxolitinib for 1 week. (E) Combined binimetinib/ruxolitinib reduced elevated reticulocytes at 1 week of treatment, superior to single-agent therapies. (F) Expanded Lin-Sca1-Kit ${ }^{+}$multipotent myeloid progenitors (MPs) were more completely reduced by combined treatment than with single agents. (G) Reduction of $\mathrm{CD} 71^{+}$erythroid progenitors was more complete with combined binimetinib/ruxolitinib than with either treatment alone. Representative FACS plots (top) and quantitation (bottom) are shown. (H) Representative FACS plots showing superior reduction of $\mathrm{Lin}^{-} \mathrm{Sca}^{-} \mathrm{Kit}^{+} \mathrm{CD} 41^{-} \mathrm{FcgR} \mathrm{R}^{-}$ CD150+CD105- megakaryocytic/erythroid progenitors (Pre-MegE) and Lin-Sca1-Kit+CD41-FcgR-CD150+CD105+ erythroid progenitors (Pre-CFU-E) by combined binimetinib/ruxolitinib. (I) Quantitation of treatment effects on myelo-erythroid progenitor populations upon 1 week of therapy. (J) Reduction of mutant allele burden as reflected by the fraction of Jak2V617F CD45.2 BM cells was superior by combined binimetinib/ruxolitinib versus single-agent therapies. Results are from recipients of Jak2V617F (CD45.2) and Jak2 wild-type (CD45.1) BM treated for 4 weeks (filled plots, $n=5$ per group) or for 1 week (open plots, $n=5-6$ per group). Quantitative results were analyzed by 1 -way ANOVA with $P \leq 0.05$ considered significant and are shown as mean \pm SD (I) or as medians with boxes representing 25 th to 75 th percentiles and whiskers indicating minimum and maximum values (B-G and J). ${ }^{*} P<0.05$, ${ }^{* *} P<0.01,{ }^{* *} P<0.005,{ }^{* * *} P<0.0001$.

FcgR-CD150+CD105- megakaryocytic-erythroid progenitors (preMegE) and Lin-Sca1-Kit ${ }^{+} \mathrm{CD} 41^{-} \mathrm{FcgR}^{-} \mathrm{CD} 15 \mathrm{O}^{+} \mathrm{CD}^{-} \mathrm{C}^{+}$erythroid progenitors (pre-CFU-E) was further improved with combined JAK/ MEK inhibition compared with either therapy alone (Figure 3, $\mathrm{H}$ and I). Combined JAK2/MEK inhibition also resulted in superior reduction of mutant allele burden as compared with JAK2 inhibitor monotherapy (Figure 3J). Findings were similar in mice with expression of the Cre recombinase under the control of the Vav (Figure 3) or the $\mathrm{Mx}-1$ promoter (Supplemental Figure 5, A-F).
BM fibrosis also substantially contributes to disease pathogenesis in MPN. We observed that MEK inhibitor monotherapy with binimetinib for 4 weeks resulted in a moderate reduction of BM and spleen fibrosis similar to ruxolitinib, although with a certain variation between individual mice. By contrast, combined ruxolitinib and binimetinib resulted in consistent resolution of fibrosis (Figure 4, A and B), suggesting that combined targeting of JAK2 and JAK2-independent MEK/ERK pathway activation is key for reversal of fibrosis. While megakaryocytic 
A

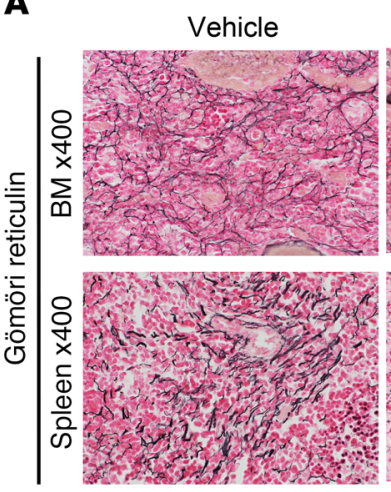

C

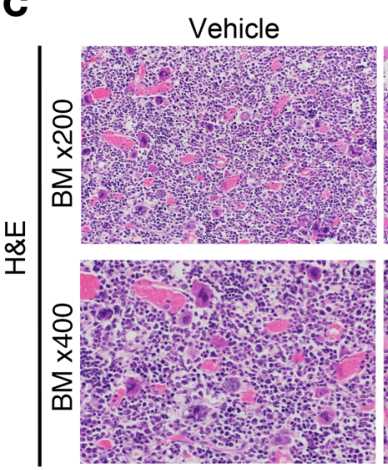

E

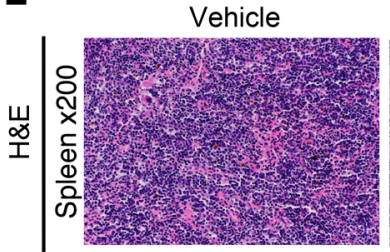

Binimetinib

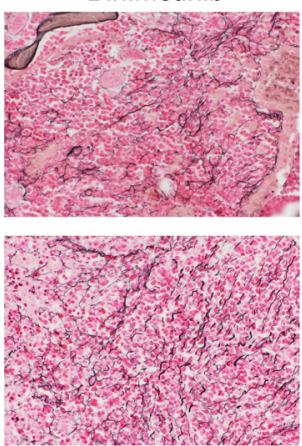

Binimetinib

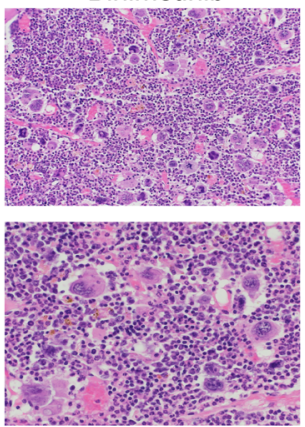

Binimetinib

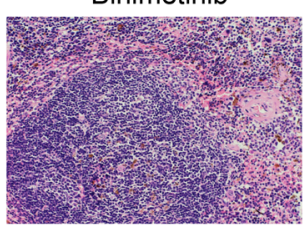

Ruxolitinib

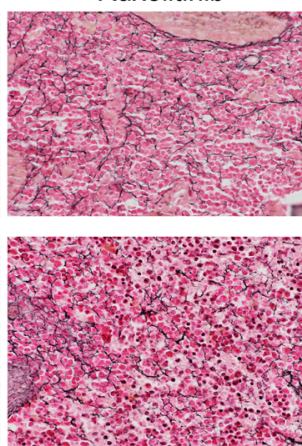

Ruxolitinib

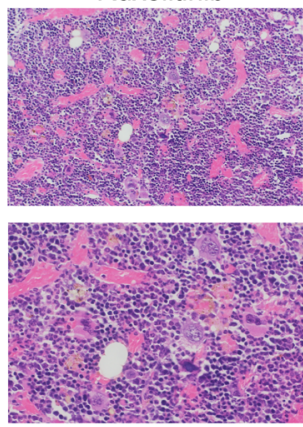

Ruxolitinib

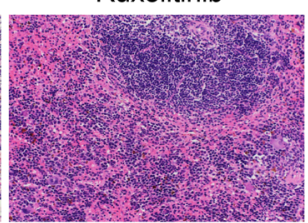

Rux + binimetinib
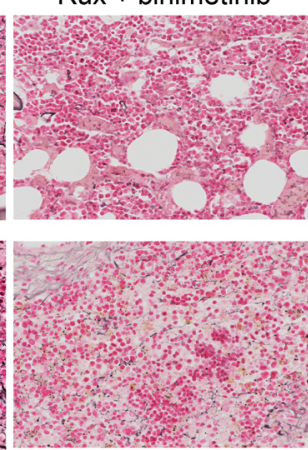

Rux + binimetinib

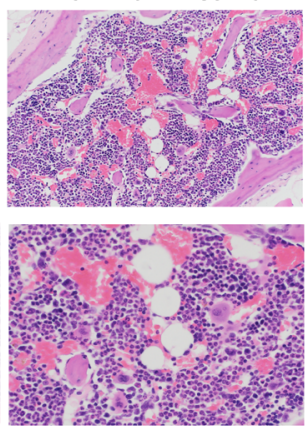

Rux + binimetinib

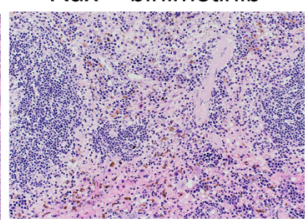

B
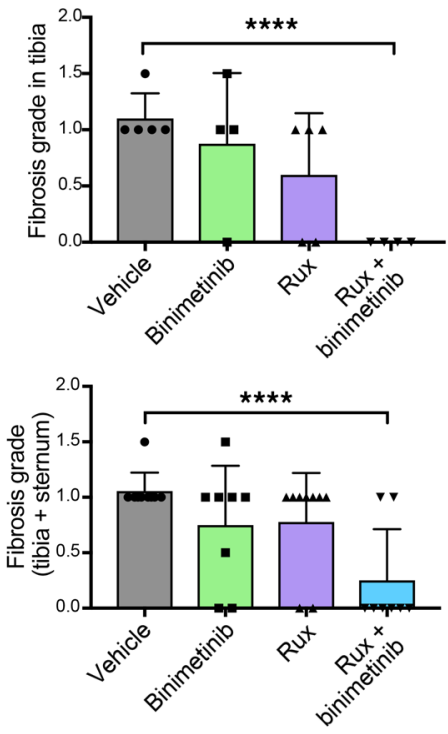

D
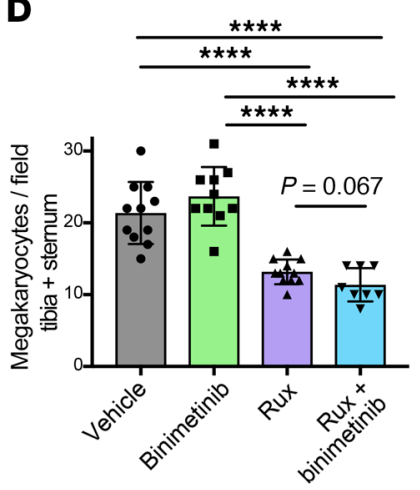

Figure 4. Combined JAK2/MEK inhibition reduces BM hypercellularity and fibrosis. (A) Gömöri reticulin staining showed superior reduction of fibrosis in BM and spleen by binimetinib and, particularly, combined binimetinib/ruxolitinib versus ruxolitinib as a single agent. (B) Quantitation of BM fibrosis according to WHO grading (myelofibrosis grade $0-3$ ) confirmed superior fibrosis reduction by combined binimetinib/ruxolitinib for 4 weeks $(n=4-5$ per group for tibia, $n=4-5$ per group for sternum). (C) H\&E staining of Jak2V617F mouse BM showed that hypercellularity was not relevantly modified by binimetinib or ruxolitinib as single agents, but was reduced by combined treatment. Megakaryocyte hyperplasia was reduced by ruxolitinib monotherapy and in combination with binimetinib, while binimetinib alone was not effective in megakaryocyte reduction. (D) Quantitation of megakaryocytes per visual field confirmed that ruxolitinib and combined ruxolitinib/binimetinib were similarly effective in megakaryocyte reduction ( $n=5$ per group in tibia, $n=3-6$ per group in sternum). (E) Myelo-erythroid infiltration of the spleen was reduced by binimetinib or ruxolitinib single-agent therapies and more effectively by combined treatment. Analyses are on recipients of Jak2V617F (CD45.2) and Jak2 wild-type (CD45.1) BM treated for 4 weeks. Quantitative results are shown as mean \pm SD and were analyzed by 1 -way ANOVA with $P \leq 0.05$ considered significant. ${ }^{* * *} P<0.001$.

hyperplasia was similarly inhibited by ruxolitinib or combined ruxolitinib/binimetinib (Figure 4, C and D), BM hypercellularity was effectively reduced by combined JAK2/MEK inhibition, but not by either treatment alone (Figure 4C). Splenic architecture was normalized from massive myelo-erythroid infiltration in vehicle-treated mice to near-physiologic follicular structure in combination therapy-treated mice (Figure 4E). Combined JAK2/MEK inhibitor treatment was well tolerated in the Jak2V617F model (Supplemental Figure 6, A-C) without increased levels of either drug when administered as combination therapy and without any significant hematologic or nonhematologic side effects (Supplemental Figure 7A). Combined JAK2/MEK inhibition was also well tolerated in C57BL/6 wild-type mice treated for the same period. Dual ruxolitinib/ binimetinib did not induce any toxicities beyond the modest cytopenias seen with ruxolitinib monotherapy in wild-type mice (Supplemental Figure 8, A-H).

Combined JAK2 and MEK inhibition has superior corrective effects on Jak2V617F-induced expression patterns. In primary (nontransplanted) Jak2V617F mice, the therapeutic benefit of combined ruxolitinib and binimetinib was also evident (Supplemental Figure 9, A-F). Congruent with reports on the MPN inflammatory milieu (55), JAK2 inhibition by ruxolitinib reduced the production of inflammatory cytokines in Jak2V617F splenocytes and BM. Notably, combined JAK2/MEK inhibition resulted in more pronounced cytokine reduction as compared with either monotherapy (Figure 5, A and B). On the protein level, cytokine levels were lowest in spleen and BM interstitial fluid and in the serum of 
Jak2V617F mice treated with combination therapy (Figure 5C and Supplemental Figure 10, A and B).

We next asked whether combined JAK/MEK inhibition would induce changes in the expression of ERK targets in vivo. Ruxolitinib as a single agent did not reduce the expression of ERK targets in Jak2V617F BM or spleen, in line with the signaling analyses demonstrating maintained ERK activation in the presence of JAK2 inhibition in MPN in vivo settings (Figure 5, D and E, and Figure 3A). Binimetinib monotherapy and combined ruxolitinib/ binimetinib significantly decreased the expression of multiple ERK targets, including the transcription factors Etv5, Egr1, and Fos, cell cycle regulators such as Ccnd1, and phosphatases including Dusp4 and Dusp6, in Jak2V617F BM and spleen, demonstrating that inhibiting ERK activation in vivo reduces MEK/ERK signaling pathway output (Figure 5, D and E). Overall, combined ruxolitinib/ binimetinib treatment induced the most extensive expression changes with respect to the expression of ERK targets and inflammatory cytokines compared with the transcriptional effects of either therapy alone (Figure 5F). Expression analysis of paired BM and spleen samples by principal component analysis (PCA) confirmed that the expression level of ERK targets was primarily determined by the treatment chosen. PCA visualized that combined ruxolitinib/binimetinib had the most pronounced effects on these parameters (Supplemental Figure 10C).

Combined JAK2 and MEK inhibition prevents ERK activation and provides superior therapeutic efficacy in an MPLW515L MPN mouse model. We next evaluated whether binimetinib is able to inhibit activation of the MEK/ERK pathway and demonstrate efficacy in the MPLW515L mutant model of MF. A single dose of $30 \mathrm{mg} / \mathrm{kg}$ binimetinib inhibited ERK activation as reflected by reduced phosphorylation in primary mouse splenocytes (Supplemental Figure 11A), and ERK inhibition was sustained with 1 and 2 weeks of treatment with binimetinib. Combined ruxolitinib and binimetinib for 2 weeks of treatment resulted in complete ERK inhibition along with reduced p-STAT3 and p-STAT5 (Figure 6A and Supplemental Figure 11B). Combined therapy was well tolerated (Supplemental Figure 6, D-F), and mice survived through the entire treatment duration, as is the case with ruxolitinib monotherapy (Figure 6B). After 2 weeks of treatment, combined ruxolitinib $60 \mathrm{mg} / \mathrm{kg}$ and binimetinib $30 \mathrm{mg} / \mathrm{kg}$ more significantly reduced splenomegaly, hepatomegaly, leukocytosis, and thrombocytosis as compared with ruxolitinib as a single agent (Figure 6, C-G). In addition, JAK/MEK inhibition reversed fibrosis to an extent not seen with JAK inhibitor monotherapy (Figure 6H). Extramedullary hematopoiesis in the liver and megakaryocytic hyperplasia of the $\mathrm{BM}$ were resolved by combined ruxolitinib and binimetinib over 2 weeks (Figure 6H). Extended treatment for 4 weeks showed analogous results (Supplemental Figure 11, C-F). Serum drug concentrations after 4 weeks of treatment showed achievement of therapeutic levels for both ruxolitinib and binimetinib without additive drug accumulation with combination therapy (Supplemental Figure 7B). Combined JAK2 and MEK inhibition with ruxolitinib and selumetinib, another non-ATP-competitive MEK1/2 inhibitor, was also effective in suppression of ERK phosphorylation and showed similar therapeutic efficacy (Supplemental Figure 12, A-D).

$M E K$ inhibition combined with type II JAK2 inhibition is also effective in a Jak2V617F model and reduces mutant allele burden.
We next assessed the efficacy of combined type II JAK2 inhibition by CHZ868 and MEK inhibition in Jak2V617F mice. Half of the usual efficacious doses administered as single agents (i.e., 15 $\mathrm{mg} / \mathrm{kg}$ for CHZ868 and binimetinib, respectively) normalized splenomegaly with 2 weeks of treatment (Supplemental Figure 13A). We observed a reduction in aberrant erythroid parameters including hematocrit and reticulocytes (Supplemental Figure 13, B and C). Notably, combined CHZ868/binimetinib reduced the expansion of $\mathrm{Lin}^{-} \mathrm{Sca} 1^{-} \mathrm{Kit}^{+}$multipotent myeloid progenitors, $\mathrm{Lin}^{-} \mathrm{Sca} 1^{-} \mathrm{Kit}^{+} \mathrm{CD} 41^{-} \mathrm{FcgR} \mathrm{CD}^{-} \mathrm{CDO}{ }^{+} \mathrm{CD} 105^{-}$megakaryocyticerythroid progenitors (pre-MegE), and $\mathrm{Lin}^{-} \mathrm{Sca} 1^{-} \mathrm{Kit}^{+} \mathrm{CD} 41^{-} \mathrm{FcgR}^{-}$ $\mathrm{CD}_{150}{ }^{+} \mathrm{CD}_{105^{+}}$erythroid progenitors (pre-CFU-E), which was not seen with CHZ868 and binimetinib when given as monotherapy at the same doses (Supplemental Figure 13, D and E). Notably, combined CHZ868/binimetinib reduced Jak2V617F mutant allele burden in mouse BM by $25 \%$ within 2 weeks (Supplemental Figure 13F). The reduction in allele burden was confirmed in $\mathrm{CD} 1^{+}$erythroid BM progenitors (Supplemental Figure 13, G and $\mathrm{H})$, suggesting that combined type II JAK2 inhibition and MEK inhibition could combine the benefits of preferential targeting of the MPN clone and prevention of JAK2-independent MEK/ERK pathway activation.

Therapeutic effects of combined JAK2/MEK inhibition are partially maintained after cessation of treatment. We next followed MPN mice after discontinuation of therapy to assess for disease recurrence when off therapy. We evaluated the evolution of the MPN phenotype after cessation of treatment in both the MPLW515L model of MF (Supplemental Figure 14, A-E) and the Jak2V617F model of PV (Supplemental Figure 15, A-F). We observed that the gross fibrosis typically seen in the MPLW515L myelofibrosis model, which responds effectively to combined JAK2/MEK inhibition (Figure $6 \mathrm{H})$, showed maintained benefit 4 weeks after stopping of the combined therapy, which was not observed with JAK2 or MEK inhibitor monotherapy (Supplemental Figure 14D). This observation was confirmed in the Jak2V617F model, which typically has less severe $\mathrm{BM}$ fibrosis. In this setting, combined JAK/MEK inhibitor treatment resulted in resolution of fibrosis (Figure 4A), which was maintained 4 weeks after cessation of combined treatment (Supplemental Figure 15F). In addition, we observed maintained therapeutic benefits for the leukocytosis and extramedullary hematopoiesis characteristic of the MPLW515L model after cessation of combined JAK2/ MEK inhibition (Supplemental Figure 14E). By contrast, we did see recurrent splenomegaly within 4 weeks of treatment cessation in both MPN models consistent with the lack of disease eradication with short-term preclinical treatment with kinase inhibitors in different disease contexts (refs. 59-63; Supplemental Figure 14, B and C; and Supplemental Figure 15, A-D).

Combined JAK2 and MEK inhibition effectively inhibits colony formation from human JAK2V617F-positive hematopoietic stem/ progenitor cells. To explore the therapeutic potential of combined JAK2 and MEK inhibition in MPN patients, we exposed hematopoietic stem/progenitor cells from MF patients to ruxolitinib, binimetinib, and combined treatment and assessed the impact on myeloid colony growth. We enriched CD $34^{+}$cells from PBMCs and let them grow in the presence of ruxolitinib, binimetinib, or both inhibitors. While JAK2 inhibition dosedependently inhibited myeloid colony formation, combined 
A

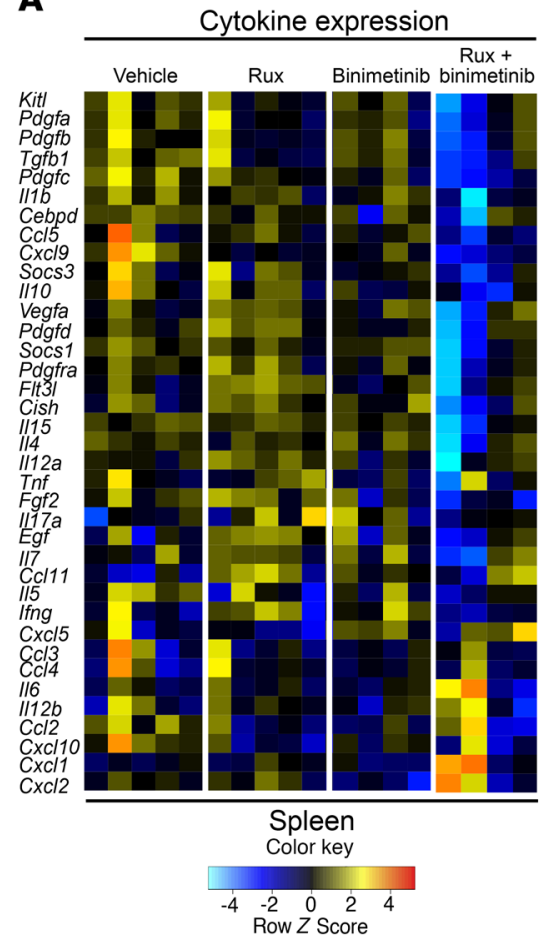

D

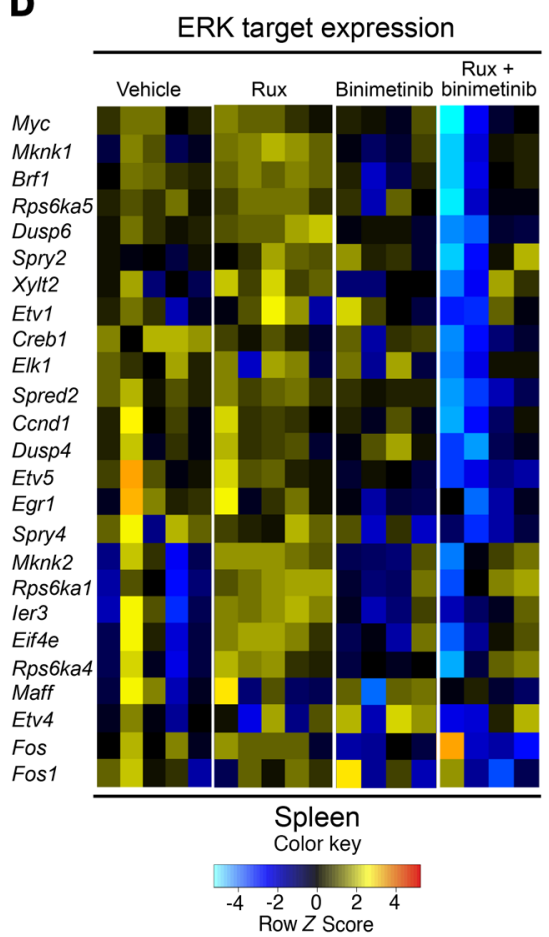

B

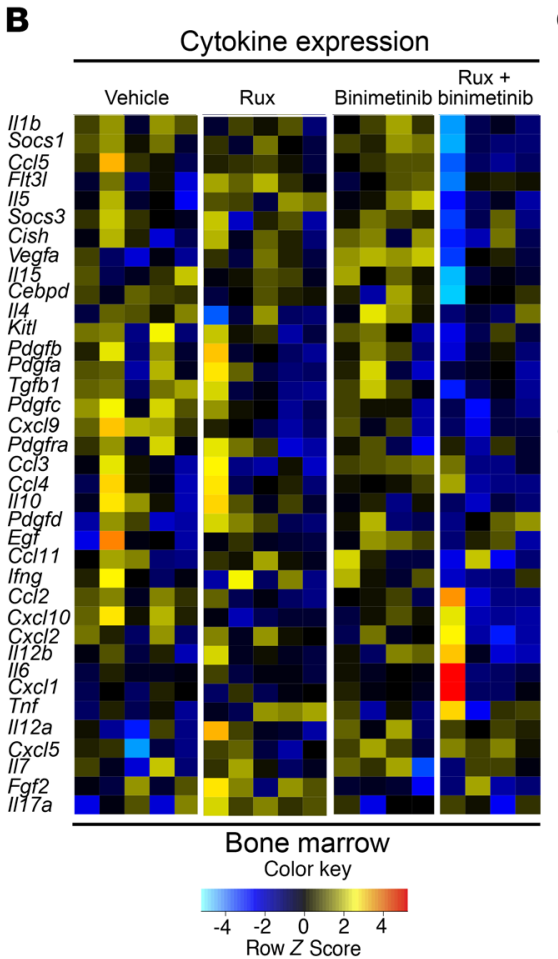

E

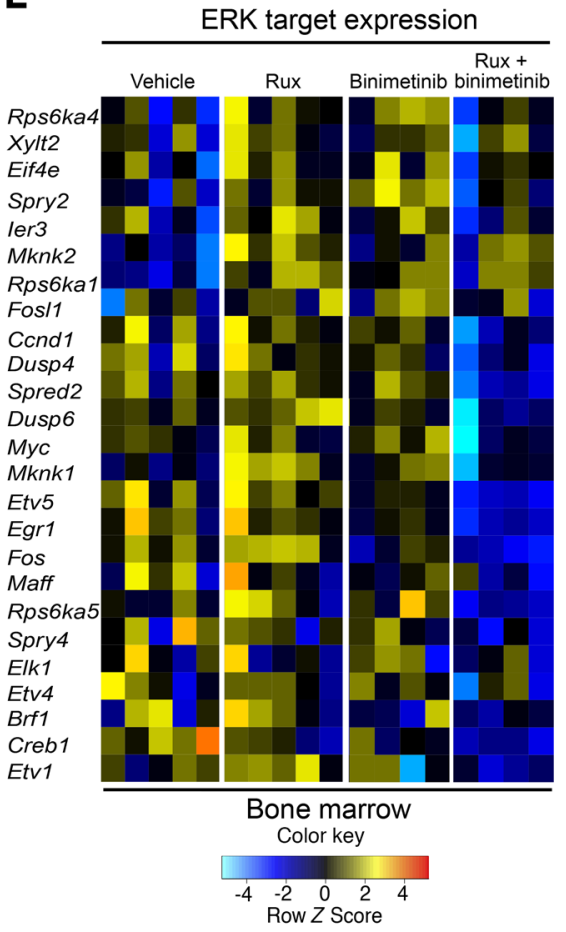

C

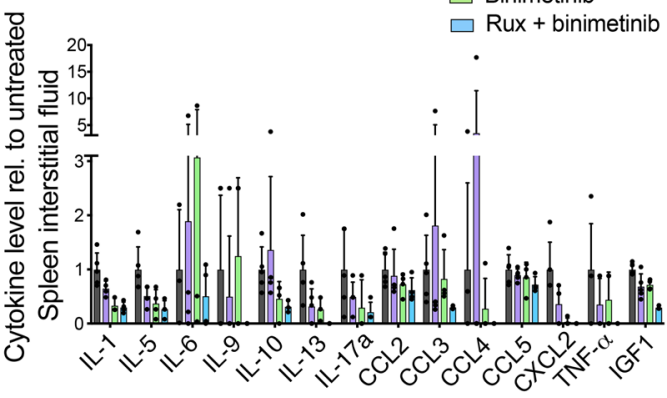

$\mathbf{F}$ - Cytokine -ERK target
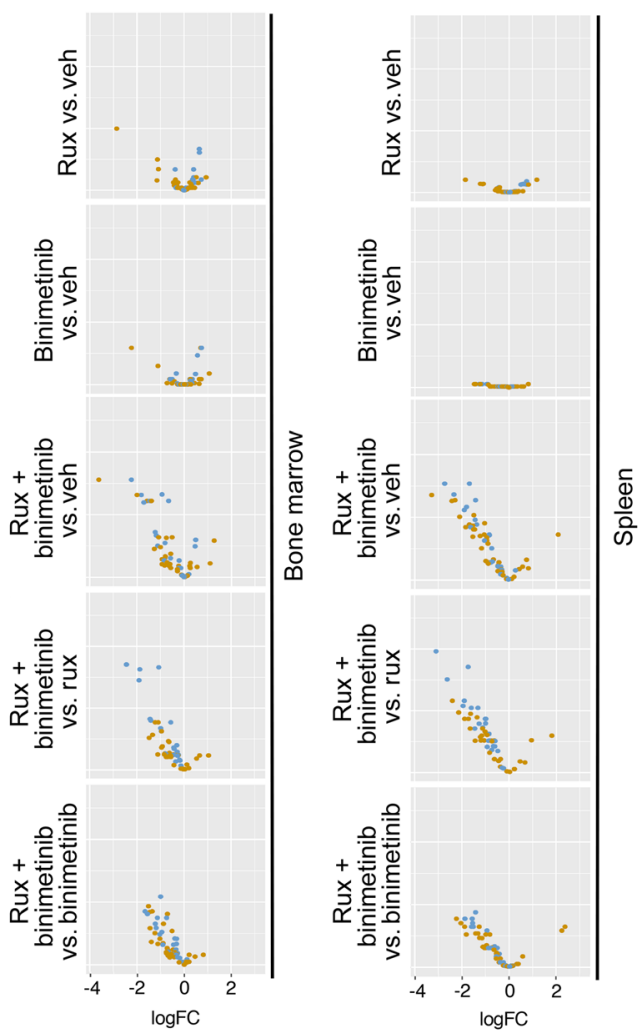

Figure 5. Combined JAK2/MEK inhibition impacts on Jak2V617F-induced expression patterns. (A) Cytokine expression was more extensively reduced by combined ruxolitinib/binimetinib than by single agents in splenocytes of Jak2V617F mice ( $n=4-5$ per group). (B) Reduction of cytokine expression was also superior by combined ruxolitinib/binimetinib in Jak2V617F BM ( $n=4-5$ per group). (C) On the protein level, several cytokines showed improved reduction in spleen interstitial fluid by combined ruxolitinib/binimetinib compared with either agent alone ( $n=3-5$ per group, $P>0.05$ by 1 -way ANOVA). (D) Combined ruxolitinib/binimetinib was superior in suppressing expression of ERK targets in Jak2V617F splenocytes ( $n=4-5$ per group). (E) Combined ruxolitinib/ binimetinib was superior in suppressing expression of ERK targets in Jak2V617F BM ( $n=4-5$ per group). (F) Volcano plots visualizing differential expression of cytokines and ERK targets in Jak2V617F BM and spleen highlight superior corrective effects of combined ruxolitinib/binimetinib ( $n=4-5$ per group). Quantitative results are shown as mean \pm SD and were analyzed by 1-way ANOVA with $P \leq 0.05$ considered significant. 
A

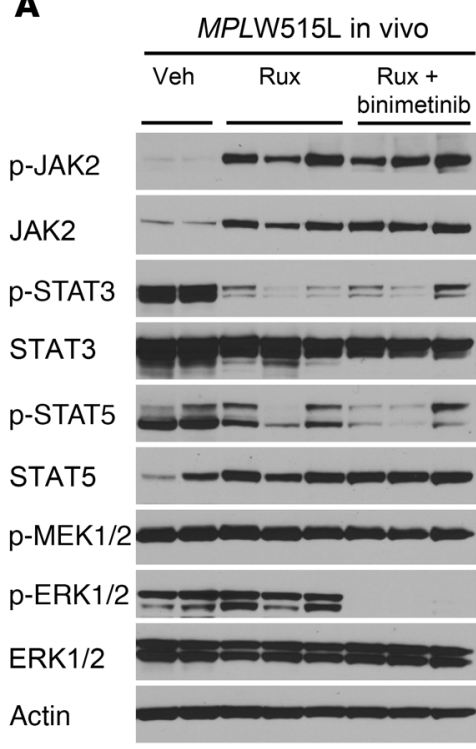

$\mathbf{F}$

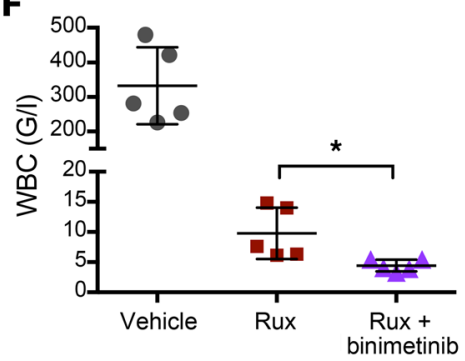

G

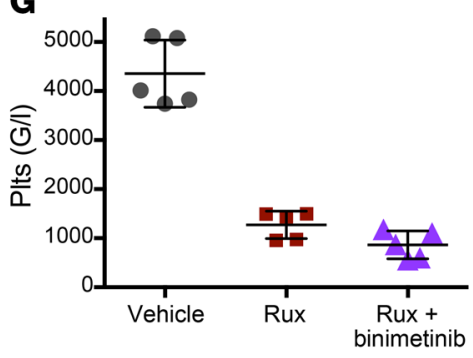

B

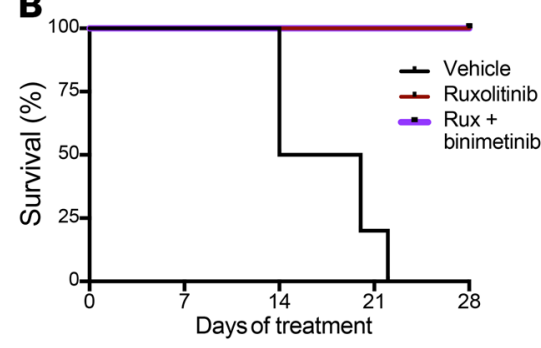

D

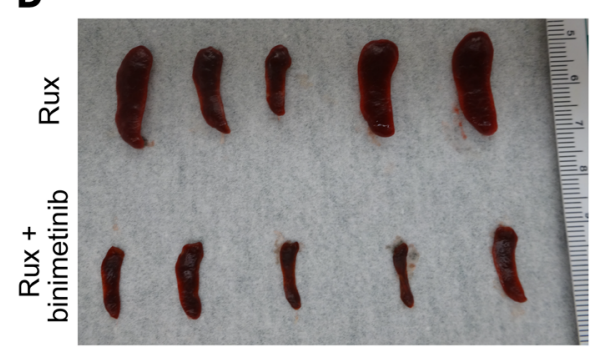

H

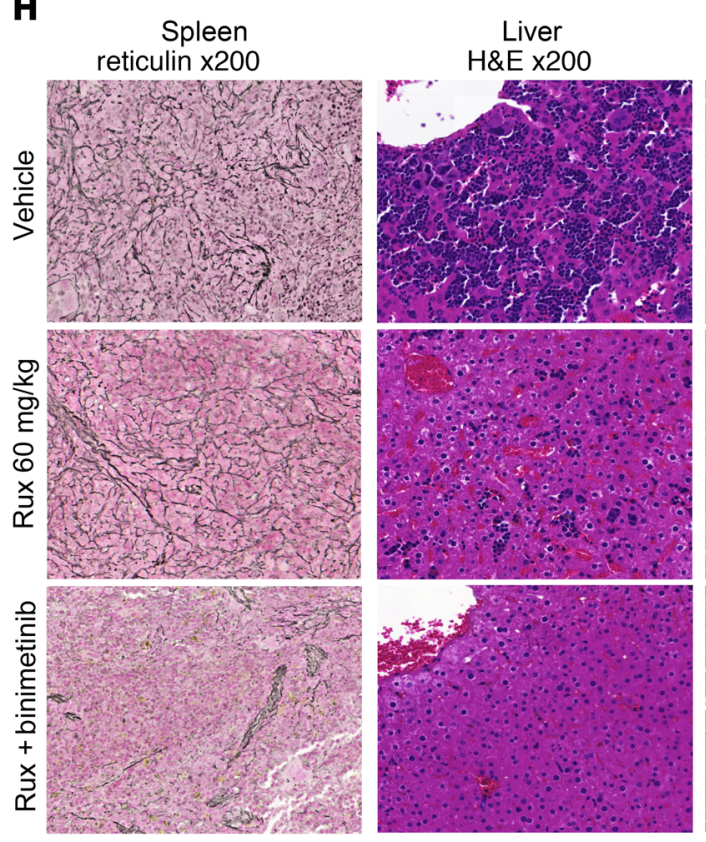

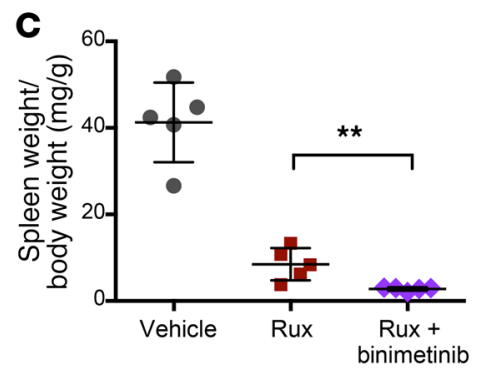

E
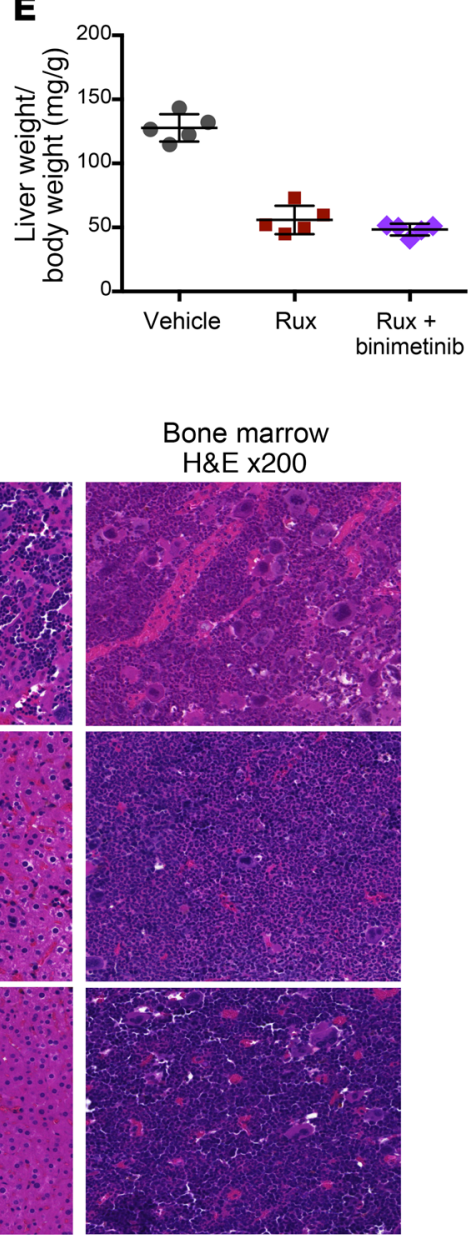

Figure 6. Combined JAK2/MEK inhibition provides superior therapeutic efficacy in an MPLW515L MPN mouse model. (A) Combined binimetinib/ruxolitinib inhibited ERK phosphorylation in MPLW515L mouse splenocytes, whereas ruxolitinib alone resulted in maintained ERK activation after 2 weeks of treatment. (B) Combined treatment was as well tolerated as ruxolitinib monotherapy, with all treated mice surviving over the entire period of the 4-week treatment study. (C-G) Combined JAK2/MEK inhibition for 2 weeks was superior to ruxolitinib monotherapy in correction of splenomegaly, hepatomegaly, leukocytosis, and thrombocytosis. 48. Plts, platelets. $\mathrm{G} / \mathrm{I}, \times 10^{9} / \mathrm{I}$. (H) Reticulin fibrosis was substantially reduced by combined JAK2/MEK inhibition as compared with ruxolitinib alone (left), as were extramedullary hematopoiesis of the liver (middle) and megakaryocytic hyperplasia of the BM (right). Results are from recipients of MPLW515L transduced BM treated for 2 weeks ( $n=5$ per group). Results from 4-week treatments are shown in Supplemental Figure 11. Quantitative results were analyzed by Student's $t$ test with $P \leq 0.05$ considered significant and are shown as mean $\pm \mathrm{SD}$. ${ }^{*} P<0.05,{ }^{* *} P<0.01$.

JAK2/MEK inhibition showed more potent inhibition at all concentrations tested. Binimetinib as a single agent significantly reduced colony number, highlighting a relevant role of MEK/ ERK pathway activation also in human MPN (Figure 7, A and B). Treatment effects were not only reflected by total colony number, but were evident from all myeloid colony subtypes, including erythroid (CFU-E, BFU-E), granulocyte-macrophage (CFU-GM), and granulocyte-erythroid-macrophage-megakaryocyte (CFU-
GEMM) colonies (Figure 7C). As MPNs are not primarily leukemic diseases with extensive circulating malignant cells, but predominantly affect the residing BM, we also exposed whole BM cells from the same patients to the respective inhibitor treatments. In line with circulating $\mathrm{CD}_{3} 4^{+}$cells, combined JAK2/MEK inhibition was superior in reduction of colony number, while both ruxolitinib and binimetinib monotherapies showed more moderate effects on all myeloid colony subtypes (Figure 7, D-G). In addition 
A

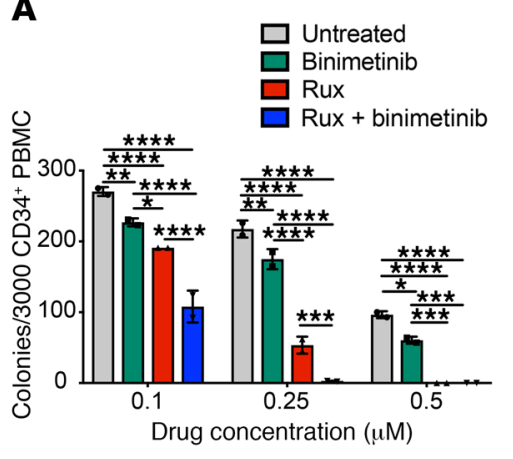

B

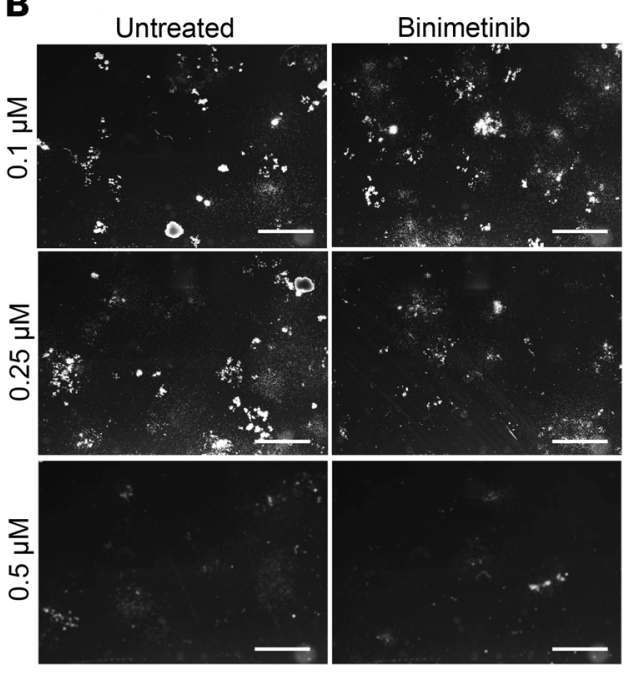

Ruxolitinib

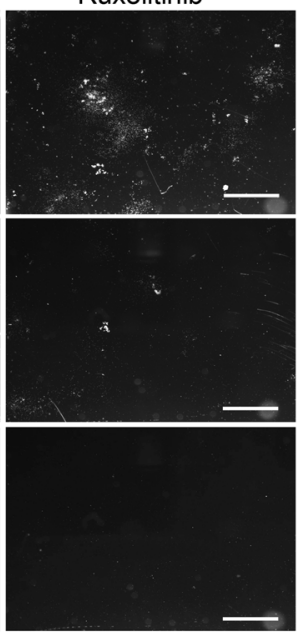

Rux + binimetinib

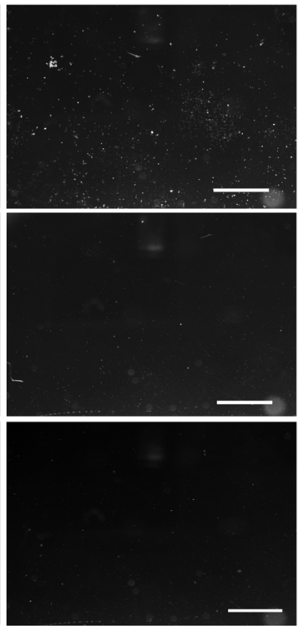

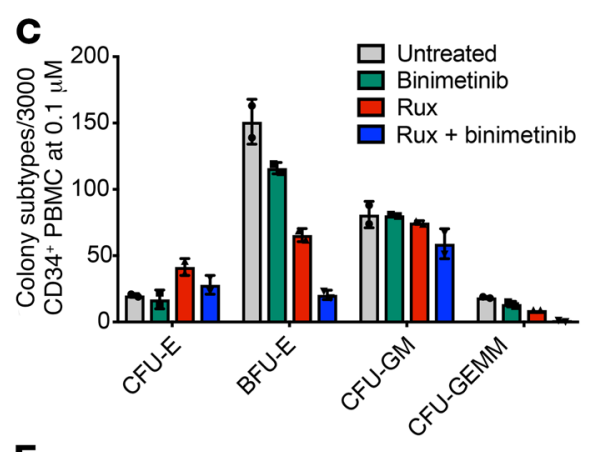

E

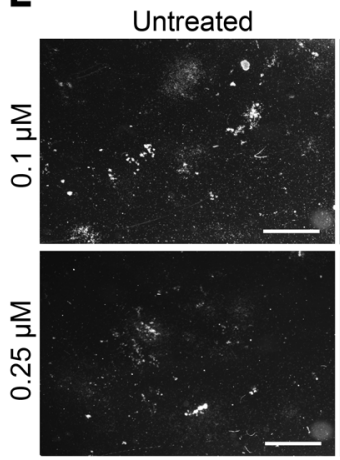

G

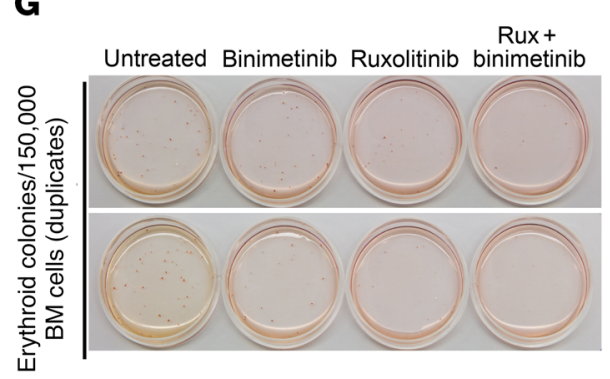

D

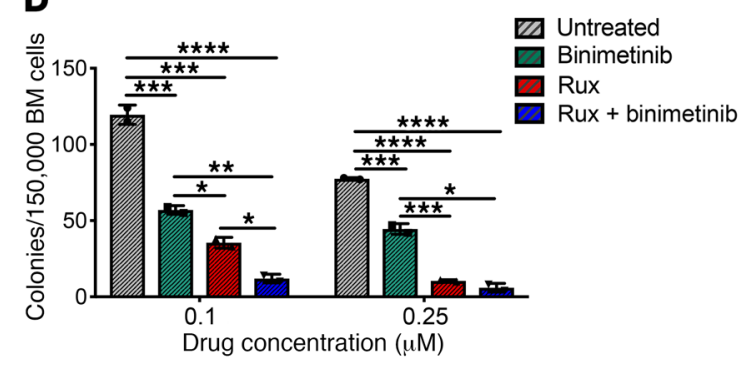

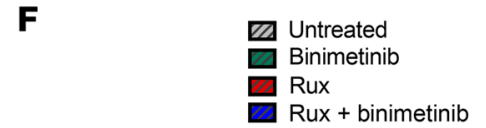

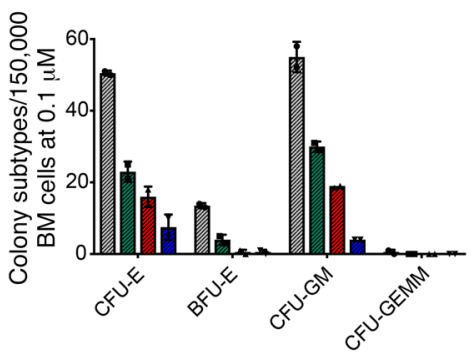

H

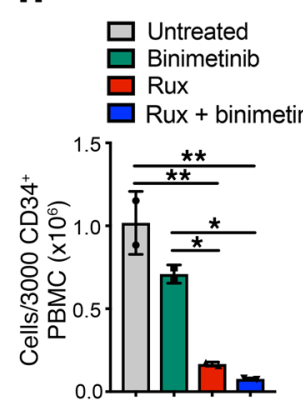

Rux + binimetinib

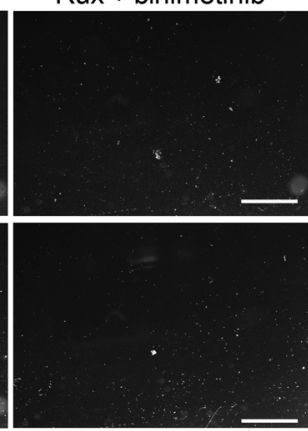

I

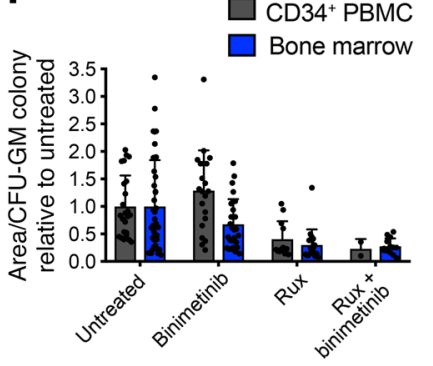


Figure 7. Combined JAK2/MEK inhibition reduces colony growth from MPN patient hematopoietic stem/progenitor cells. Paired PBMC and BM samples from JAK2V617F MF patients were assessed $(n=4)$. Results of a representative patient are shown in duplicate. Untreated samples were exposed to respective amounts of DMSO. (A and B) Combined ruxolitinib/binimetinib reduced total colony numbers from $\mathrm{CD}_{3} 4^{+}$stem/ progenitor cells enriched from PBMCs to a greater extent than singleagent therapies. (C) Colony subtypes including erythroid (CFU-E, BFU-E), granulocyte-macrophage (CFU-GM), and granulocyte-erythroidmacrophage-megakaryocyte (CFU-GEMM) from CD34+ cells showed a superior response to combined JAK2/MEK inhibition. (D and E) Total colony numbers from whole BM cells were reduced to a greater extent by combined ruxolitinib/binimetinib than by single agents. ( $\mathbf{F}$ and $\mathbf{G}$ ) Colony subtypes including erythroid (CFU-E, BFU-E) and granulocytemacrophage (CFU-GM) from whole BM showed a superior response to combined JAK2/MEK inhibition, as illustrated by a photograph of native erythroid colonies. ( $\mathbf{H}$ and $\mathbf{I})$ Cell numbers per well and the size of the colonies were more effectively reduced by combined ruxolitinib/ binimetinib than by single agents. Quantitative results are shown as mean \pm SD and were analyzed by 1-way ANOVA with $P \leq 0.05$ considered significant. ${ }^{*} P<0.05,{ }^{*} P<0.01,{ }^{* *} P<0.005$, ${ }^{* * *} P<0.001$.

to reduced colony numbers, combined ruxolitinib/binimetinib also resulted in formation of smaller colonies as reflected by the area per colony (Figure 7, $\mathrm{H}$ and I).

\section{Discussion}

The finding of activated JAK2 signaling as a central feature in MPN led to the development of JAK inhibitors (4), such that ruxolitinib is now the first molecularly targeted therapy and standard of care for patients with MF and refractory PV $(1,64,65)$. Type I JAK2 inhibition by ruxolitinib reduces splenomegaly and constitutional symptoms and prolongs survival, which represent important therapeutic benefits for MPN patients. However, ruxolitinib cannot eliminate the MPN clone when given as monotherapy, suggesting limited disease-modifying potential (26-28). In addition, development of resistance has been described, which leads to a loss of the clinical response in a substantial fraction of patients $(30,33,66)$. It is therefore key to gain insight into the molecular events that interfere with effective targeting of the malignant clone in MPN. Here we show that MEK/ERK signaling can serve as a survival signal in MPN, and that targeting the oncogenic signaling network at several nodes can increase efficacy in MPN therapy.

In MPN, we observed maintained activation of the MEK/ERK signaling pathway in vivo in the presence of JAK2 inhibitors. While ERK activation is JAK2-dependent in MPN cell lines (Figure 1A) as reported (12), ERK phosphorylation persisted upon type I or type II JAK2 inhibition in vivo. Our data demonstrate that MEK/ERK pathway activation in MPN in vivo is relevant to MPN pathogenesis and treatment, as we observe a superior therapeutic effect by combined JAK2 and MEK/ERK pathway inhibition. Beyond activated MEK/ERK signaling in MPN, the MEK/ERK pathway is critical in many cancers, including hematologic malignancies $(34,35)$, and targeting oncogenic MEK/ERK signaling has proven beneficial in melanoma, thyroid cancer, lung, and colorectal cancer, hairy cell leukemia, and KRAS/NRAS-mutated leukemias (38). In many of these malignancies, components of the MEK/ERK pathway itself such as RAF or RAS, or upstream tyrosine kinase receptors, harbor somatic mutations and mediate constitutive activation (67). In
MPN, we found activated MEK/ERK signaling, despite effective targeting of the JAK2 oncogene, suggesting compensatory signaling bypassing JAK2 inhibition.

By 2 complementary screening approaches, we identified PDGF/ PDGFR $\alpha$ signaling as a mediator of JAK2-independent MEK/ERK pathway activation in MPN. Notably, PDGFR $\alpha$ has long been known to be involved in the pathogenesis of other myeloid malignancies, such as chronic eosinophilic leukemia, but unlike in MPN, chromosomal translocations involving PDGFR $\alpha$ mediate constitutive activation of PDGFR $\alpha$ signaling in those settings (68). By contrast, our data support the notion of the PDGF-BB-PDGFR $\alpha$ ligand-receptor pair as a mediator of compensatory signaling, which bypasses JAK2 inhibition and mediates JAK2-independent ERK activation in MPN in vivo. Our studies suggest that PDGF-BB is the primary ligand mediating this effect, with higher levels of Pdgfb expression in hematopoietic tissues and higher PDGF-BB concentrations in serum, BM, and spleen as compared with PDGF-AA. As both ligands are known to activate PDGFR $\alpha$, the relative functional roles of PDGF-AA and PDGF-BB deserve further studies in MPN in vivo including primary samples. Furthermore, other secreted cytokines may play an additional role, which will need to be studied in subsequent investigations in patient samples and preclinical models.

PDGF has long been implicated in the development of BM fibrosis in MPN along with TGF- $\beta$ and bFGF $(57,69,70)$. Mainly produced by megakaryocytes, PDGF is primarily stored and released from $\alpha$ granules in megakaryocytes and platelets, but production in fibroblasts, osteoblasts, and endothelial cells has also been delineated (57). In an overexpression mouse model of thrombopoietin (Tpo), which signals through JAK2, previous studies implicated PDGF and TGF- $\beta$ in mediating fibrosis (71). Thus, a role of PDGF in promoting the phenotype of MPNs, which are driven by activated JAK2 signaling, is concordant with these findings. In addition, it has been shown that the expression of PDGFA, $P D G F B$, and PDGFRA is increased in the BM of MF patients as compared with healthy individuals, and this upregulation was particularly pronounced for PDGFB and PDGFRA (72). PDGFRA was primarily expressed in $\mathrm{BM}$ megakaryocytes of these patients and not in fibroblasts, suggesting an autocrine or paracrine activation loop by PDGF on the hematopoietic cells in MPNs. Here we show that in MPNs, PDGF-BB is a mediator of BM fibrosis along with other fibrogenic cytokines and activates the MEK/ ERK signaling pathway independent of JAK2 in MPN models and human MPN cell lines. Our results from combined JAK2 and MEK inhibition demonstrate that the signaling from the PDGFBPDGFR $\alpha$ ligand-receptor pair has a critical role in MPN as a JAK2independent driver of the entire MPN phenotype. It will be important to see whether this signaling pathway is relevant in other myeloid malignancies, and whether it can mediate resistance to targeted therapies in other malignant contexts.

Combined targeting of JAK2 and MEK by ruxolitinib and binimetinib inhibited ERK activation and showed increased therapeutic efficacy. The therapeutic efficacy of combined JAK2/MEK inhibition was superior in multiple aspects of MPN phenotype correction, including erythrocytosis, leuko- and thrombocytosis, BM hypercellularity, megakaryocytic hyperplasia, and progenitor cell expansion, in the Jak2V617F and MPLW515L mutant MPN models. Combined JAK2/MEK inhibition was particularly effective in 
reversal of fibrosis and reduced the degree of fibrosis to an extent not seen with previous therapies in MPN. Based on our results and previous evidence, we hypothesize that the mechanism mediating reduced fibrosis could be 2-fold: suppression of megakaryocyte hyperplasia leading to reduced production and release of PDGF, and inhibition of PDGF-induced MEK/ERK pathway activation in fibroblasts of the BM niche. Further studies will be needed in order to dissect the molecular processes by which PDGF and PDGFR isoforms mediate auto- and paracrine effects on the MPN clone as well as on the cellular environment in the niche (73).

Overall, we describe here a new aspect of oncogenic signaling in MPN that is mediated through compensatory, cell-extrinsic MEK/ ERK pathway activation, and we show that dual targeting of JAK2 and MEK provides improved therapeutic efficacy by preventing bypass ERK activation. Our studies also highlight the importance of assessing sensitivity and resistance to targeted therapies in the in vivo setting, as this observation would not have emanated from cell line pharmacologic and/or functional genomic approaches. Most importantly, our findings suggest that dual JAK/MEK inhibition should be pursued as a therapeutic approach for MPN patients. Our data also suggest that compensatory MEK/ERK pathway activation might represent a mechanism of resistance to molecularly targeted therapies that is only observed in the in vivo setting, and which can guide mechanism-based combination therapy studies in different malignant contexts.

\section{Methods}

Supplemental Methods are available online with this article; https:// doi.org/10.1172/JCI98785DS1.

Inhibitors. Ruxolitinib for in vitro and ex vivo applications was purchased from Chemietek and stored at $1 \mathrm{mM}$ in DMSO at $-20^{\circ} \mathrm{C}$. For in vivo use, ruxolitinib was administered orally at 60 or $90 \mathrm{mg} /$ $\mathrm{kg}$ twice daily (b.i.d.). Binimetinib was purchased from Chemietek or Sellekchem and administered orally at $30 \mathrm{mg} / \mathrm{kg}$ b.i.d. Selumetinib was from Chemietek and was administered orally at $25 \mathrm{mg} / \mathrm{kg}$ b.i.d. CHZ868 was synthesized by Novartis and administered orally at 15 $\mathrm{mg} / \mathrm{kg}$ once daily. Inhibitor doses for combined treatment in vivo were chosen based on dose finding studies (binimetinib) or empirically (ruxolitinib, CHZ868).

Signaling analyses in MPN cells and mouse models. SET- 2 cells originally obtained from ATCC were cultured in RPMI 1640/20\% FBS and incubated for 4 hours at $10^{6}$ cells $/ \mathrm{ml}$ with inhibitor or DMSO, washed in ice-cold PBS, and collected in lysis buffer supplemented with Protease Arrest (EMD) and Phosphatase Inhibitor Cocktail II (Calbiochem). Total protein was normalized by Bio-Rad Bradford quantitation and separated on $4 \%-12 \%$ Bis-Tris gels (Invitrogen), and blots were probed for p-ERK1/2 (clone D13.14.4E, catalog 4370), ERK1/2 (clone 137F5, catalog 4695), p-MEK1/2 (clone 41G9, catalog 9154), MEK1/2 (clone 47E6, catalog 9126), p-STAT3 (clone D3A7, catalog 9145), STAT3 (clone 79D7, catalog 4904), p-STAT5 (clone C11C5, catalog 9359), p-JAK2 (clone C80C3, catalog 3776), JAK2 (clone D2E12, catalog 3230), PDGFR $\alpha$ (clone D1E1E, catalog 3174), actin (clone 13E5, catalog 4970), and GAPDH (clone D4C6R, catalog 97166) from Cell Signaling Technology; STAT5 (catalog sc-835, C-17) from Santa Cruz Biotechnology; and p-PDGFR $\alpha$ (clone EP2478, catalog ab134068) from Abcam. For analysis of signaling after in vivo inhibitor treatment, mice were sacrificed 4 hours (ruxolitinib, binimetinib, selumetinib) or
2 hours (CHZ868) after the last oral dose. Splenocyte single-cell suspensions were directly lysed as described above, and bone marrow (BM) cells were boiled in SDS sample buffer followed by electrophoresis and Western blotting. For analysis of signaling after ex vivo inhibitor exposure, BM and spleen cells from untreated mice were serumstarved for 1 hour in RPMI 1640/1\% BSA and exposed to 0.5-2 $\mu \mathrm{M}$ ruxolitinib for 6-8 hours followed directly by lysis as described above. NIH Image J software was used for densitometries of band intensities.

Analysis of MPN patient samples. The Memorial Sloan Kettering Cancer Center institutional review board and the Ethical Committee Basel approved sample collection and experiments. Informed consent was available from all patients. For signaling analyses, PBMCs were freshly isolated by Ficoll centrifugation, serum-starved for 1 hour in $\alpha \mathrm{MEM} / 1 \%$ BSA, and exposed to 0.5-2 $\mu \mathrm{M}$ ruxolitinib or DMSO for 16 hours ex vivo, followed by lysis and Western blotting as described above.

For colony formation assays, paired peripheral blood and BM samples were obtained from JAK2V617F MF patients $(n=4)$. CD34 ${ }^{+}$ stem/progenitor cells were enriched from PBMCs using human CD34 microbeads (Miltenyi Biotec) and plated in duplicate at 3000 cells per well into MethoCult media (STEMCELL Technologies, 04435). Whole BM cells were plated in duplicate both at 100,000 and at 150,000 cells per well into MethoCult media (STEMCELL Technologies, 04034). MethoCult was supplemented with ruxolitinib, binimetinib, or both inhibitors at $0.1,0.25$, and $0.5 \mu \mathrm{M}$. For untreated conditions, MethoCult was supplemented with respective amounts of DMSO. Number and types of colonies were scored after 15 days. Images were taken on an Olympus SZX12 microscope.

RTK arrays in MPN mouse models. Jak2V617F mice provided by A. Mullally, Harvard University (Cambridge, Massachusetts, USA), or $M P L W 515 \mathrm{~L}$ mice were treated with ruxolitinib or vehicle for 5 days and sacrificed 4 hours after the last dose. Receptor tyrosine kinase (RTK) arrays were incubated with splenocyte lysate at 500-650 $\mu$ g total protein and were developed according to the manufacturer's instructions. To account for variable background intensities of individual arrays, signal intensities within each array were ranked and rankings compared across arrays.

PDGF stimulation in MPN mouse models. Jak2V617F BM and spleen cells from untreated mice were serum-starved for 1 hour in RPMI 1640/1\% BSA followed by stimulation with $100 \mathrm{ng} / \mathrm{ml}$ mPDGF-AA (PeproTech) for 10 minutes or $200 \mathrm{ng} / \mathrm{ml} \mathrm{mPDGF-BB}$ (PeproTech) for 5 minutes as previously described (74-77) in the presence of $1 \mu \mathrm{M}$ ruxolitinib ex vivo. Untreated cells were exposed to respective amounts of DMSO. Alternatively, primary Jak2V617F BM or spleen cells were pretreated with $1 \mu \mathrm{M}$ ruxolitinib in RPMI 1640/1\% BSA for 6 hours followed by stimulation as described.

Jak2V617F and MPLW515L MPN mouse models. Primarily, a conditional Jak2V617F knockin model of PV was used (53), which is characterized by Jak2V617F expression in hematopoietic tissues under the control of the Vav or the Mx-1 promoter. For in vivo signaling studies (Figure 1), primary Jak2V617F Vav-Cre mice received 3-5 oral doses of inhibitor. For ex vivo signaling studies including PDGF stimulation experiments (Figures 1 and 2), untreated primary Jak2V617F Vav-Cre mice were used as described above. For treatment studies, BM from primary Jak2V617F Vav-Cre CD45.2 mice was mixed 1:1 with Jak2 wild-type CD45.1 BM and transplanted into lethally irradiated CD45.1 recipients. Development of the MPN phenotype was confirmed by peripheral blood counts 2 months after BM transplantation. Mice were 
randomized to treatment groups according to blood counts and treated by oral gavage for 1-4 weeks (Figures 3 and 4; and Supplemental Figures 13 and 15). The same setup was used with BM from primary Jak2V617F Mx-1-Cre mice for confirmation of the treatment effects in the context of Mx-1-Cre, and randomized mice were treated for 1 week (Supplemental Figure 5). For studies of tumor recurrence, treatment was followed by a 4-week observation period off therapy (Supplemental Figures 14 and 15). For analysis of Jak2V617F-induced expression patterns, Jak2V617F Vav-Cre mice were used after being treated with respective inhibitors for 9 days (Figure 5 and Supplemental Figure 9). As a model of MPLW515L mutant MPN, CD117-enriched (Miltenyi Biotec) BALB/c BM was transduced with retroviral supernatant containing MSCV- $h M P L W 515 L-I R E S-G F P$ and injected into lethally irradiated BALB/c recipients $(14,54)$. Development of the MPN phenotype was confirmed by blood counts 3 weeks after BM transplantation. For in vivo signaling analyses, MPLW515L mice received 3-5 doses of inhibitor (Figure 1 and Supplemental Figure 1E). For ex vivo signaling studies, untreated MPLW515L mice were used as outlined above (Figure $1 \mathrm{H}$ and Supplemental Figure $1 \mathrm{G}$ ). For treatment studies, mice were randomized to treatment groups according to blood counts and treated by oral gavage for 1-4 weeks (Figure 6 and Supplemental Figures 11, 12 , and 14). For histopathology, tissues were fixed in $4 \%$ paraformaldehyde, paraffin-embedded, and stained with H\&E. Gömöri stain was used for assessment of reticulin fibers. Fibrosis was graded by a specialized hematopathologist. Images were taken on an Olympus BX43 using cellSens software version 1.6.

Animal care was in strict compliance with institutional guidelines established by Memorial Sloan Kettering Cancer Center, the Guide for the Care and Use of Laboratory Animals (National Academies Press, 2011), and the Association for Assessment and Accreditation of Laboratory Animal Care International. All animal procedures were in accordance with the Guide for the Care and Use of Laboratory Animals and were approved by the Institutional Animal Care and Use Committees at Memorial Sloan Kettering Cancer Center. Animal care and all animal procedures and experiments were also in strict adherence to Swiss laws for animal welfare and were approved by the Swiss Cantonal Veterinary Office of Basel-Stadt.

Flow cytometry for myelo-erythroid progenitor populations and mutant allele burden. Flow cytometry analyses were performed in the Jak2V617F model in recipients of 1:1 mixed Jak2V617F CD45.2 and Jak2 wild-type CD45.1 BM. BM cells were stained with antibodies for lineage markers including B220 (clone RA3-6B2, catalog 103236), CD11b (clone M1/70, catalog 108427), Gr-1 (clone RB6-8C5, catalog 108427), CD4 (clone RM4-4, catalog 116012), CD8 (clone 53-6.7, catalog 100733), CD127 (clone A7R34, catalog 135022), and CD49b (clone DX5, catalog 108915) and for Sca-1 (clone D7, catalog 108105), c-Kit (clone 2B8, catalog 135135), CD41 (clone MWReg30, catalog 133905), CD150 (clone TC15-12F12.2, catalog 115903), CD48 (clone HM48-1, catalog 103411), CD16/32 (clone 93, catalog 101314), CD71 (clone RI7217, catalog 113805), and Ter-119 (clone TER-119, catalog 116211) from BioLegend and CD105 (clone MJ7/18, catalog 48-1051-82) from eBioscience as previously described (78) and for CD45.1 (clone A20, catalog 110721) and CD45.2 (clone 104, catalog 109827) from BioLegend to assess mutant allele burden as the fraction of CD45.2/CD45 total BM or erythroid progenitor cells. Analyses were performed on an LSRFortessa cell analyzer (BD).

RNA expression analyses by NanoString and quantitative real-time $P C R$. RNA was extracted from BM and spleen of treated Jak2V617F mice using the Nucleospin RNA Plus kit (Macherey-Nagel). For cDNA synthesis, 500 ng of RNA was reverse-transcribed with the HighCapacity cDNA Reverse Transcription Kit (Applied Biosystems). Expression levels of several cytokines and known ERK targets were analyzed using the NanoString platform (79) according to the manufacturer's hybridization protocol for nCounter XT CodeSet Gene Expression assays with $100 \mathrm{ng}$ total RNA input per sample. Final data collection was on an nCounter Digital Analyzer. Raw data were normalized using 6 housekeeping genes, from which the subset with the lowest variance across samples was determined after averaging of the log-transformed read counts. The variance was determined by the median of variances of 1000 bootstrap replicates. Normalized values were calculated by averaging out of log-transformed read counts of selected housekeeping genes and subtraction from log-transformed read counts of target genes. Heatmaps were created using the gplots package in $\mathrm{R}$, version 3.4.0, and row dendrograms using the correlation distance metric. Differential expression was calculated by the limma Bioconductor package (80). For volcano plots, ggplot2 R package was used. Quantitative real-time PCR on an ABI Quantstudio ViiA7 was used to validate expression of $P d g f a, P d g f b$, and Pdgfra in Jak2V617F BM and spleen in triplicates using a SYBR Green PCR Master Mix kit (Applied Biosystems). For expression analysis in specific BM and spleen cell populations, RNA was processed analogously with the Quick-RNA Microprep kit (Zymo Research) from populations sorted on a FACSAria (BD) including $\mathrm{Lin}^{-} \mathrm{Sca}{ }^{+} \mathrm{C}-\mathrm{Kit}^{+}$(LSK) cells, $\mathrm{Lin}^{-} \mathrm{Sca1}^{-} \mathrm{C}^{-} \mathrm{Kit}^{+} \mathrm{FcgR}^{\mathrm{lo}} \mathrm{CD} 34^{+}$common myeloid progenitors (CMPs), $\mathrm{Lin}^{-} \mathrm{Sca1}{ }^{-} \mathrm{c}^{-} \mathrm{Kit}^{+} \mathrm{FcgR}^{\mathrm{lo}} \mathrm{CD} 34^{-}$megakaryocyte-erythroid progenitors (MEPs), Lin $^{-} \mathrm{Sca1}^{-} \mathrm{c}-\mathrm{Kit}^{+} \mathrm{FcgR}^{\text {hi }}{ }^{-\mathrm{CD} 34^{+}}$granulocyte-monocyte progenitors (GMPs), Mac- $1^{+} \mathrm{Gr}-1^{+}$granulocytes, $\mathrm{Mac}-1^{+} \mathrm{Gr}-1^{-}$monocytes, and $\mathrm{B}_{22} \mathrm{O}^{+} \mathrm{B}$ and $\mathrm{CD}^{+} \mathrm{T}$ lymphocytes of Jak2V617F mice. Antibodies were as specified above and against CD34 (clone RAM34, catalog 11-034182, eBioscience) and CD3 (clone 17A2, catalog 100205, BioLegend). Primers are listed in Supplemental Table 1.

Cytokine analyses by Luminex in MPN mouse models. Jak2V617F mice treated for 9 days were euthanized by $\mathrm{CO}_{2}$ inhalation and bled by cardiac puncture. Blood was allowed to clot at $37^{\circ} \mathrm{C}$ for 1 hour and centrifuged at $1016 \mathrm{~g}$ for 10 minutes to collect serum. BM interstitial fluid was collected by flushing of bones into $1 \mathrm{ml}$ of PBS, followed by filtration through $70-\mu \mathrm{m}$ filters (Falcon). Spleens were homogenized, filtered, and spun down in $2 \mathrm{ml}$ PBS. Sera, BM, and spleen interstitial fluid were snap-frozen and kept at $-80^{\circ} \mathrm{C}$. Cytokines/growth factors in $\mathrm{BM}$ and spleen interstitial fluid were measured by 28-plex and 3-plex magnetic custom mix Luminex kits (R\&D) and analyzed on a Bio-Plex 200 system (Bio-Rad). Concentrations were calculated from standard curves by Bio-Plex 200 Manager 5.0 software.

Statistics. Results are shown as mean \pm SD or SEM. To assess statistical significance between groups, 1-way ANOVA with Bonferroni post hoc multiple-comparisons testing or 2-tailed unpaired Student's $t$ test was used (GraphPad Prism version 7). $P$ values of 0.05 or less were considered statistically significant.

Study approval. Experiments in preclinical animal models were approved by the Institutional Animal Care and Use Committees at Memorial Sloan Kettering Cancer Center, New York, New York, USA, as well as by the Cantonal Veterinary Office Basel-Stadt, Basel, Switzerland. Informed consent was obtained from MPN patients whose clinical isolates were analyzed, and respective studies were approved by the institutional review board of Memo- 
rial Sloan Kettering Cancer Center and by the Ethical Committee Basel, Basel, Switzerland.

\section{Author contributions}

SCM and RLL designed the study and wrote the paper. SCM, SS, TC, SB, AB, NG, HHS, SD, MSD, CS, BAK, MK, SC, MK, MH, ASB, JRP, TR, and RCS performed experiments, analyzed data, or provided essential experimental materials. All authors edited and approved the manuscript.

\section{Acknowledgments}

We thank Ann Mullally for Jak2V617F mice and Dario Sterker, Christine Mann-Ran, Beat Erne, Lifen Xu, and Ronny Nienhold for technical support. This work was supported by research grants from the Swiss National Science Foundation (PBBEP3_144806, PZO0P3_161145), Swiss Cancer League/Swiss Cancer Research (KFS-3005-08-2012, KFS-3858-02-2016), Foundation for the Fight against Cancer, Foundation Peter-Anton and Anna-Katharina Miescher and the Swiss Society of Hematology, the Swiss BRIDGE
Foundation, the Nora van Meeuwen-Häfliger Foundation, and the Huggenberger-Bischoff Foundation for Cancer Research to SCM; National Cancer Institute grants P01 CA108671 and R35197594 to RLL; NIH grant K99 HL122503-01A1 to M. Kleppe; the Janus Project to RLL; and a Leukemia \& Lymphoma Society Specialized Center of Research grant to RLL. Studies supported by Memorial Sloan Kettering core facilities were supported in part by Memorial Sloan Kettering Cancer Center Support Grant/Core Grant P30 CA008748. M. Kleppe is a fellow of the American Society of Hematology.

Address correspondence to: Sara C. Meyer, Division of Hematology, Department of Medicine and Department of Biomedicine, University Hospital Basel, Petersgraben 4, 4031 Basel, Switzerland. Phone: 41.61.556.59.65; Email: sara.meyer@unibas.ch. Or to: Ross L. Levine, Human Oncology and Pathogenesis Program, Leukemia Service, Department of Medicine, Memorial Sloan Kettering Cancer Center, 1275 York Avenue, Box 20, New York, New York 10065, USA. Phone: 646.888.2767; Email: leviner@mskcc.org.
1. Spivak JL. Myeloproliferative neoplasms. N Engl J Med. 2017;376(22):2168-2181.

2. Gangat N, et al. DIPSS plus: a refined Dynamic International Prognostic Scoring System for primary myelofibrosis that incorporates prognostic information from karyotype, platelet count, and transfusion status. J Clin Oncol. 2011;29(4):392-397.

3. Jain T, Mesa RA, Palmer JM. Allogeneic stem cell transplantation in myelofibrosis. Biol Blood Marrow Transplant. 2017;23(9):1429-1436.

4. Rampal R, et al. Integrated genomic analysis illustrates the central role of JAK-STAT pathway activation in myeloproliferative neoplasm pathogenesis. Blood. 2014;123(22):e123-e133.

5. Hobbs GS, Rozelle S, Mullally A. The development and use of Janus kinase 2 inhibitors for the treatment of myeloproliferative neoplasms. Hematol Oncol Clin North Am. 2017;31(4):613-626.

6. Neubauer H, Cumano A, Müller M, Wu H, Huffstadt U, Pfeffer K. Jak2 deficiency defines an essential developmental checkpoint in definitive hematopoiesis. Cell. 1998;93(3):397-409.

7. Parganas E, et al. Jak2 is essential for signaling through a variety of cytokine receptors. Cell. 1998;93(3):385-395.

8. Meyer SC, Levine RL. Molecular pathways: molecular basis for sensitivity and resistance to JAK kinase inhibitors. Clin Cancer Res. 2014;20(8):2051-2059.

9. Kralovics R, et al. A gain-of-function mutation of JAK2 in myeloproliferative disorders. N Engl J Med. 2005;352(17):1779-1790.

10. Levine RL, et al. Activating mutation in the tyrosine kinase JAK2 in polycythemia vera, essential thrombocythemia, and myeloid metaplasia with myelofibrosis. Cancer Cell. 2005;7(4):387-397.

11. Baxter EJ, et al. Acquired mutation of the tyrosine kinase JAK2 in human myeloproliferative disorders. Lancet. 2005;365(9464):1054-1061.

12. James $\mathrm{C}$, et al. A unique clonal JAK2 mutation leading to constitutive signalling causes polycythaemia vera. Nature. 2005;434(7037):1144-1148.
13. Scott LM, et al. JAK2 exon 12 mutations in polycythemia vera and idiopathic erythrocytosis. $N$ Engl JMed. 2007;356(5):459-468.

14. Pikman Y, et al. MPLW515L is a novel somatic activating mutation in myelofibrosis with myeloid metaplasia. PLoS Med. 2006;3(7):e270.

15. Nangalia J, et al. Somatic CALR mutations in myeloproliferative neoplasms with nonmutated JAK2. N Engl J Med. 2013;369(25):2391-2405.

16. Klampfl T, et al. Somatic mutations of calreticulin in myeloproliferative neoplasms. $\mathrm{N} \mathrm{Engl} \mathrm{JMed.}$ 2013;369(25):2379-2390.

17. Marty C, et al. Calreticulin mutants in mice induce an MPL-dependent thrombocytosis with frequent progression to myelofibrosis. Blood. 2016;127(10):1317-1324.

18. Chachoua I, et al. Thrombopoietin receptor activation by myeloproliferative neoplasm associated calreticulin mutants. Blood 2016;127(10):1325-1335.

19. Elf S, et al. Mutant calreticulin requires both its mutant C-terminus and the thrombopoietin receptor for oncogenic transformation. Cancer Discov. 2016;6(4):368-381.

20. Harrison C, et al. JAK inhibition with ruxolitinib versus best available therapy for myelofibrosis. N Engl JMed. 2012;366(9):787-798.

21. Verstovsek S, et al. A double-blind, placebocontrolled trial of ruxolitinib for myelofibrosis. N Engl J Med. 2012;366(9):799-807.

22. Vannucchi AM, et al. Ruxolitinib versus standard therapy for the treatment of polycythemia vera. N Engl J Med. 2015;372(5):426-435.

23. Verstovsek S, et al. A phase 2 study of momelotinib, a potent JAK1 and JAK2 inhibitor, in patients with polycythemia vera or essential thrombocythemia. Leuk Res. 2017;60:11-17.

24. Mesa RA, et al. Pacritinib versus best available therapy for the treatment of myelofibrosis irrespective of baseline cytopenias (PERSIST-1):an international, randomised, phase 3 trial. Lancet Haematol. 2017;4(5):e225-e236.
25. Andraos R, et al. Modulation of activation-loop phosphorylation by JAK inhibitors is binding mode dependent. Cancer Discov. 2012;2(6):512-523.

26. Cervantes F, et al. Three-year efficacy, safety, and survival findings from COMFORT-II, a phase 3 study comparing ruxolitinib with best available therapy for myelofibrosis. Blood. 2013;122(25):4047-4053.

27. Verstovsek $S$, et al. Long-term treatment with ruxolitinib for patients with myelofibrosis: 5-year update from the randomized, double-blind, placebo-controlled, phase 3 COMFORT-I trial. J Hematol Oncol. 2017;10(1):55.

28. Verstovsek S, et al. Long-term survival in patients treated with ruxolitinib for myelofibrosis: COMFORT-I and -II pooled analyses. J Hematol Oncol. 2017;10(1):156.

29. Verstovsek S, et al. Efficacy, safety and survival with ruxolitinib in patients with myelofibrosis:results of a median 2-year follow-up of COMFORT-I. Haematologica. 2013;98(12):1865-1871.

30. Ellis MH, Koren-Michowitz M, Lavi N, Vannucchi AM, Mesa R, Harrison CN. Ruxolitinib for the management of myelofibrosis: results of an international physician survey. Leuk Res. 2017;61:6-9.

31. Koppikar P, et al. Heterodimeric JAK-STAT activation as a mechanism of persistence to JAK2 inhibitor therapy. Nature. 2012;489(7414):155-159.

32. Meyer SC, et al. CHZ868, a type II JAK2 inhibitor, reverses type IJAK inhibitor persistence and demonstrates efficacy in myeloproliferative neoplasms. Cancer Cell. 2015;28(1):15-28.

33. Meyer SC. Mechanisms of resistance to JAK2 inhibitors in myeloproliferative neoplasms. Hematol Oncol Clin North Am. 2017;31(4):627-642.

34. Chang L, Karin M. Mammalian MAP kinase signalling cascades. Nature. 2001;410(6824):37-40.

35. Chung E, Kondo M. Role of Ras/Raf/MEK/ERK signaling in physiological hematopoiesis and leukemia development. Immunol Res. 2011;49 (1-3):248-268.

36. Chan G, Gu S, Neel BG. Erk1 and Erk2 are required for maintenance of hematopoietic 
stem cells and adult hematopoiesis. Blood. 2013;121(18):3594-3598.

37. Staser K, et al. Normal hematopoiesis and neurofibromin-deficient myeloproliferative disease require Erk. J Clin Invest. 2013;123(1):329-334.

38. Santarpia L, Lippman SM, El-Naggar AK. Targeting the MAPK-RAS-RAF signaling pathway in cancer therapy. Expert Opin Ther Targets. 2012;16(1):103-119.

39. Meyer SC, Levine RL. Translational implications of somatic genomics in acute myeloid leukaemia. Lancet Oncol. 2014;15(9):e382-e394.

40. Tiacci E, et al. BRAF mutations in hairy-cell leukemia. N Engl JMed. 2011;364(24):2305-2315.

41. García-Ortí L, et al. Integration of SNP and mRNA arrays with microRNA profiling reveals that MiR-370 is upregulated and targets NF1 in acute myeloid leukemia. PLoS One. 2012;7(10):e47717.

42. Lyubynska N, et al. A MEK inhibitor abrogates myeloproliferative disease in Kras mutant mice. Sci Transl Med. 2011;3(76):76ra27.

43. Grimaldi AM, Simeone E, Festino L, Vanella V, Strudel M, Ascierto PA. MEK inhibitors in the treatment of metastatic melanoma and solid tumors. Am J Clin Dermatol. 2017;18(6):745-754.

44. Tiacci E, Pettirossi V, Schiavoni G, Falini B. Genomics of hairy cell leukemia. JClin Oncol. 2017;35(9):1002-1010.

45. Tiacci E, et al. Targeting mutant BRAF in relapsed or refractory hairy-cell leukemia. N Engl JMed. 2015;373(18):1733-1747.

46. Flaherty KT, et al. Inhibition of mutated, activated BRAF in metastatic melanoma. N Engl J Med. 2010;363(9):809-819.

47. Long GV, et al. Combined BRAF and MEK inhibition versus BRAF inhibition alone in melanoma. N Engl JMed. 2014;371(20):1877-1888.

48. Abdel-Wahab O, et al. Efficacy of intermittent combined RAF and MEK inhibition in a patient with concurrent BRAF- and NRAS-mutant malignancies. Cancer Discov. 2014;4(5):538-545.

49. Lito P, et al. Relief of profound feedback inhibition of mitogenic signaling by RAF inhibitors attenuates their activity in BRAFV600E melanomas. Cancer Cell. 2012;22(5):668-682.

50. Durrant DE, Morrison DK. Targeting the Raf kinases in human cancer: the Raf dimer dilemma. Br JCancer. 2018;118(1):3-8.

51. Shang J, Lu S, Jiang Y, Zhang J. Allosteric modulators of MEK1: drug design and discovery. Chem Biol Drug Des. 2016;88(4):485-497.

52. Wolf A, et al. JAK2-V617F-induced MAPK activity is regulated by PI3K and acts synergistically with PI3K on the proliferation of JAK2-V617F-positive cells. JAKSTAT. 2013;2(3):e24574.

53. Mullally A, et al. Physiological Jak2V617F expression causes a lethal myeloproliferative neoplasm with differential effects on hematopoietic stem and progenitor cells. Cancer Cell. 2010;17(6):584-596.

54. Koppikar P, et al. Efficacy of the JAK2 inhibitor INCB16562 in a murine model of MPLW515Linduced thrombocytosis and myelofibrosis. Blood. 2010;115(14):2919-2927.

55. Kleppe M, et al. JAK-STAT pathway activation in malignant and nonmalignant cells contributes to MPN pathogenesis and therapeutic response. Cancer Discov. 2015;5(3):316-331.

56. Tefferi A, Vaidya R, Caramazza D, Finke C, Lasho T, Pardanani A. Circulating interleukin (IL)-8, IL-2R, IL-12, and IL-15 levels are independently prognostic in primary myelofibrosis: a comprehensive cytokine profiling study. JClin Oncol. 2011;29(10):1356-1363.

57. Demoulin JB, Montano-Almendras CP. Plateletderived growth factors and their receptors in normal and malignant hematopoiesis. Am J Blood Res. 2012;2(1):44-56.

58. Akinleye A, Furqan M, Mukhi N, Ravella P, Liu D. MEK and the inhibitors: from bench to bedside. J Hematol Oncol. 2013;6:27.

59. Cohen NA, Kim TS, DeMatteo RP. Principles of kinase inhibitor therapy for solid tumors. Ann Surg. 2017;265(2):311-319.

60. Saikia T. The cure of chronic myeloid leukemia: are we there yet? Curr Oncol Rep. 2018;20(2):12.

61. Cheloni G, et al. Targeting chronic myeloid leukemia stem cells with the hypoxia-inducible factor inhibitor acriflavine. Blood. 2017;130(5):655-665.

62. Kim TS, et al. Increased KIT inhibition enhances therapeutic efficacy in gastrointestinal stromal tumor. Clin Cancer Res. 2014;20(9):2350-2362.

63. Burke RT, et al. A potential therapeutic strategy for chronic lymphocytic leukemia by combining Idelalisib and GS-9973, a novel spleen tyrosine kinase (Syk) inhibitor. Oncotarget. 2014;5(4):908-915.

64. Cervantes F. How I treat myelofibrosis. Blood. 2014;124(17):2635-2642.

65. Vannucchi AM. How I treat polycythemia vera. Blood. 2014;124(22):3212-3220.

66. Andreoli A, et al. Clinical resistance to ruxolitinib is more frequent in patients without MPN-associated mutations and is rarely due to mutations in the JAK2 kinase drug-binding domain. Blood. 2013;122(21):1591.

67. Davies $\mathrm{H}$, et al. Mutations of the BRAF gene in human cancer. Nature. 2002;417(6892):949-954.

68. Reiter A, Gotlib J. Myeloid neoplasms with eosinophilia. Blood. 2017;129(6):704-714.

69. Hasselbalch HC, et al. Imatinib mesylate in idiopathic and postpolycythemic myelofibrosis. Am J Hematol. 2003;74(4):238-242.

70. Desterke C, Martinaud C, Ruzehaji N, Le Bousse-Kerdilès MC. Inflammation as a keystone of bone marrow stroma alterations in primary myelofibrosis. Mediators Inflamm. 2015;2015:415024.

71. Yan XQ, et al. A model of myelofibrosis and osteosclerosis in mice induced by overexpressing thrombopoietin (mpl ligand): reversal of disease by bone marrow transplantation. Blood. 1996;88(2):402-409.

72. Bock O, Loch G, Büsche G, von Wasielewski R, Schlué J, Kreipe H. Aberrant expression of plateletderived growth factor (PDGF) and PDGF receptoralpha is associated with advanced bone marrow fibrosis in idiopathic myelofibrosis. Haematologica. 2005;90(1):133-134.

73. Heldin CH. Autocrine PDGF stimulation in malignancies. Ups J Med Sci. 2012;117(2):83-91.

74. Seikrit C, et al. Biological responses to PDGF-AA versus PDGF-CC in renal fibroblasts. Nephrol Dial Transplant. 2013;28(4):889-900.

75. Enarsson M, Erlandsson A, Larsson H, Forsberg-Nilsson K. Extracellular signalregulated protein kinase signaling is uncoupled from initial differentiation of central nervous system stem cells to neurons. Mol Cancer Res. 2002;1(2):147-154.

76. Cao R, et al. PDGF-BB induces intratumoral lymphangiogenesis and promotes lymphatic metastasis. Cancer Cell. 2004;6(4):333-345.

77. Fingas CD, et al. Myofibroblast-derived PDGFBB promotes Hedgehog survival signaling in cholangiocarcinoma cells. Hepatology. 2011;54(6):2076-2088.

78. Pronk CJ, et al. Elucidation of the phenotypic, functional, and molecular topography of a myeloerythroid progenitor cell hierarchy. Cell Stem Cell. 2007;1(4):428-442.

79. Geiss GK, et al. Direct multiplexed measurement of gene expression with color-coded probe pairs. Nat Biotechnol. 2008;26(3):317-325.

80. Ritchie ME, et al. limma powers differential expression analyses for RNA-sequencing and microarray studies. Nucleic Acids Res. 2015;43(7):e47. 